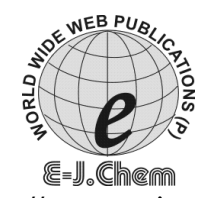

http://www.e-journals.net

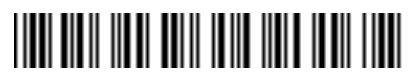

ISSN: 0973-4945; CODEN ECJHAO

E-Journal of Chemistry

Vol. 4, No. 3, pp. 320-342, July 2007

\title{
Synthesis, Characterization and Pharmacological Activities of 3,6-Disubstituted-1,2,4-triazolo [3,4-b]- 1,3,4-thiadiazoles and their Dihydro Analogues
}

\author{
VINOD MATHEW*, J. KESHAVAYYA and V. P. VAIDYA \\ *Dept. of Pharmaceutical Chemistry, \\ Acharya \& B. M. Reddy College of Pharmacy, \\ Bangalore-560090, Karnataka, India. \\ Department of Studies in Chemistry, Kuvempu University, \\ Jnana Sahayadri, Shankaraghatta- 577451, Shimoga, Karnataka, India.
}

Received 16 November 2006; Accepted 16 December 2006

\begin{abstract}
Amino-5-aryl/heteroaryl substituted-3-mercapto-1,2,4triazoles 3(a-d) were prepared from the corresponding aromatic carboxylic acids through a multi-step sequence. Compounds 3(a-d) were made to react with various aromatic/hetero aromatic acids and hetero aromatic aldehydes to give 3,6-disubstituted-1,2,4-triazolo [3,4-b]-1,3,4-thiadizoles and 3,6-disubstituted-5,6-dihydro-1,2,4triazolo [3,4-b]-1,3,4-thiadizoles respectively. Elemental analysis, IR, ${ }^{1} \mathrm{H}$ NMR and mass spectral data elucidated the structures of all newly synthesized compounds. Synthesized compounds are studied for their antibacterial, antifungal, anti-inflammatory and analgesic activities. Some of the tested compounds showed significant pharmacological activities.
\end{abstract}

Keywords: Triazoles, Triazolothiadiazoles, Dihydro triazolothiadiazoles, Pharmacological activities.

\section{Introduction}

1,2,4-Triazole and 1,3,4-thiadiazoles represent one of the most biologically active classes of compounds, possessing a wide spectrum of activities ${ }^{1-5}$. Various substituted 1,2,4-triazolo $[3,4-b]-1,3,4$-thiadiazoles and their dihydro analogues are associated with diverse pharmacological activities such as antimicrobial ${ }^{6}$, antibacterial ${ }^{7}$, antitubercular ${ }^{8}$, antiinflammatory $^{9-10}$, antifungal ${ }^{11}$ etc. A triazolo thiadiazole system may be viewed as a cyclic 
analogue of two very important components thiosemicarbazide ${ }^{12}$ and biguanide ${ }^{13}$, which often display diverse biological activities. Prompted by these observations, as part of our research program aimed at developing new biologically active nitrogen and sulphur containing heterocycles, we report the synthesis of some new 3,6-disubstituted-1,2,4triazolo[3,4-b]-1,3,4-thiadiazoles (Scheme 2) and their 5,6-dihydro analogues (Scheme 3). 4-Amino-3-aryl/aralkyl/heteroaryl substituted-5-mercapto-1,2,4-triazole 3(a-d) were prepared using the method ${ }^{14}$ (Scheme 1). The structures of the intermediate triazole derivatives were based on their elemental analysis and other spectral data.

Condensation of the triazoles 3 (a-d) with aromatic acids in the presence of phosphorous oxychloride (Scheme 2) produced a series of triazolo thiadiazoles (4-16); while its condensation with hetero aromatic aldehydes (Scheme 3 ) afforded a series of 5,6-dihydro triazolo thiadiazoles (17-20). The structure assigned to compounds was substantiated by their analytical and other spectral data.

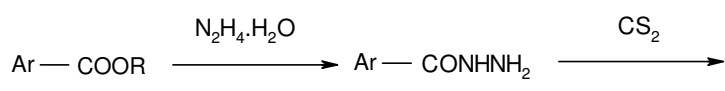

(1) $\mathrm{KOH} / \mathrm{C}_{2} \mathrm{H}_{5} \mathrm{OH}$<smiles>N[AlH2]c1nnc(S)n1N</smiles>

(2)

Scheme 1<smiles>[R]c1cc(C(=O)O)c2ccccc2n1</smiles><smiles>[R]c1cc(C(=O)O)cc([2H])n1</smiles>

7-9(a-d)

Scheme 2

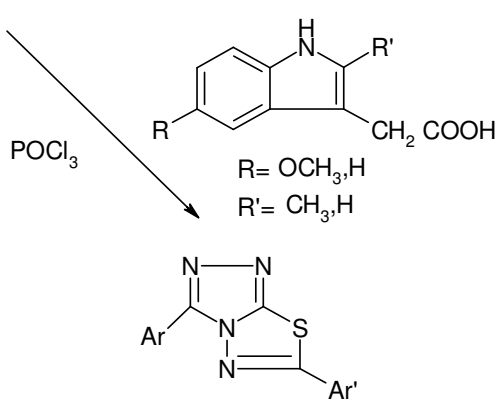

$10-12(a-d)$ 


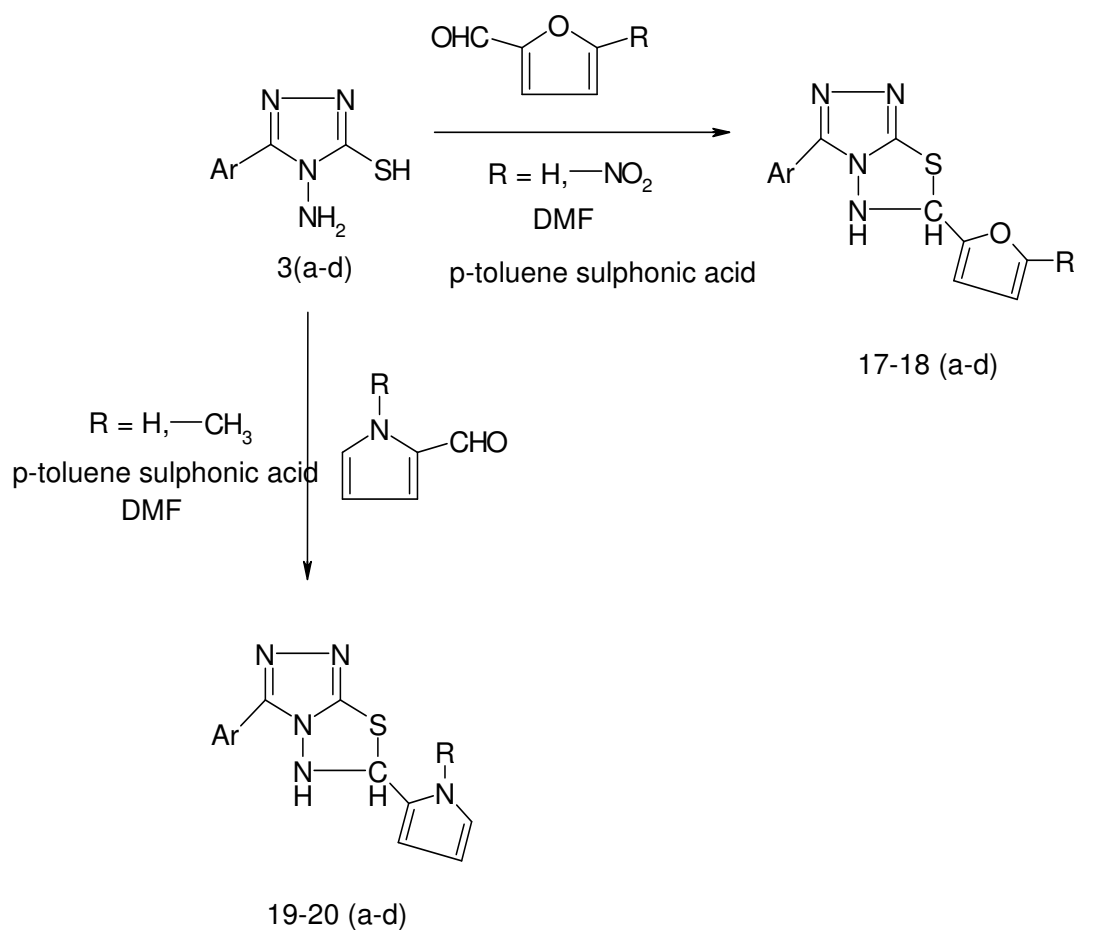

\section{Experimental}

Scheme 3

Thin layer chromatography was used to access the completion of the reaction and purity of the compounds synthesized. Melting points were taken in open glass capillary tubes using thiels tube containing liquid paraffin and are uncorrected. IR spectra in $\mathrm{KBr}$ were recorded on a Shimadzu-8400 FTIR spectrophotometer, ${ }^{1} \mathrm{H}$ NMR spectra were recorded on Brucker spectrophotometer $(400 \mathrm{MHz})$ in $\mathrm{DMSO}-\mathrm{d}_{6} / \mathrm{CDCl}_{3}$ using TMS as an internal standard (chemical shifts are expressed in $\delta \mathrm{ppm}$ ), mass spectra were recorded in Finnigan MAT 8230 mass spectrophotometer and elemental analysis were recorded on Thermo Finnigan FLASH EA 1112 CHNS anlyser. The purity of the compounds were checked on silica gel-G coated plates by using ethyl acetate and petroleum ether (1:1) as the eluent and observed in UV light. All the synthesized compounds gave satisfactory elemental analyses.

\section{General procedure for the preparation of Aryl hydrazide. (1)}

To the methyl/ethyl esters of substituted aromatic acids $(0.1 \mathrm{~mol})$, hydrazine hydrate $(0.1 \mathrm{~mol})$ was added and refluxed the solution for $30 \mathrm{~min} .20 \mathrm{~mL}$ of ethanol was added to the refluxing mixture as a solvent in order to homogenize solution. The resulting mixture was further allowed to reflux for $6 \mathrm{~h}$. Excess ethanol was distilled out and the contents were allowed to cool. The crystals formed was filtered, washed thoroughly with water and dried. The completion of the reaction was monitored on TLC by using silica gel-G coated plates by using ethyl acetate and petroleum ether (1:1) as the eluent and observed under UV light. 
General procedure for the preparation of 2-aryl substituted-5-mercapto-1,3,4oxadiazole (2)

To a solution of $1(0.1 \mathrm{~mol})$ in ethanol $(30 \mathrm{~mL}), \mathrm{KOH}(0.1 \mathrm{~mol})$ in absolute ethanol $(50 \mathrm{~mL})$ and $\mathrm{CS}_{2}(0.2 \mathrm{~mol})$ were added and refluxed for about $5 \mathrm{~h}$ till evolution of hydrogen sulfide was ceased. The reaction mixture was cooled at room temperature and diluted with water. On acidification with dilute hydrochloric acid, the required oxadiazoles was precipitated. It was filtered, thoroughly washed with cold water and recrystallised from ethanol.

General procedure for the preparation of 3-substituted-4-amino-5-mercapto-1,2, 4triazole.3 $(a-d)$

A mixture of $2(0.1 \mathrm{~mol})$ and hydrazine hydrate $(0.1 \mathrm{~mol})$ in dry pyridine $(15 \mathrm{~mL})$ was refluxed for about $4 \mathrm{~h}$. The reaction mixture was cooled at room temperature and was neutralized with dilute hydrochloric acid. The solid obtained was filtered, thoroughly washed with cold water and recrystallised from ethanol.

\section{4-Amino-3- (3,4-dimethoxy phenyl) -5- mercapto-1,2,4-triazole.(3a)}

Yield: 60\%; m.p: $212{ }^{\circ} \mathrm{C}$; IR (KBr) $\vee\left(\mathrm{cm}^{-1}\right): 3291$ (NH stretching), 1613 (C=N stretching); 3130 (aromatic CH stretching), $2586(\mathrm{SH}), 2934,2840$ (methyl CH stretch), 1269 (asymmetric C-O-C stretching), 1021 (symmetric C-O-C stretching), $1284(\mathrm{~N}-\mathrm{N}=\mathrm{C}), 1582,1552,1479(\mathrm{C}=\mathrm{C}$ ring stretching); ${ }^{1} \mathrm{H}$ NMR $\delta$ (ppm): 13.8 (s, 1H, SH), 7.1 (d,1H, C-5 of Ar), 7.56 (s, 1H, C-2 of Ar), 7.7 (d,1H, C-6 of Ar), $5.78\left(\mathrm{~s}, 2 \mathrm{H}, \mathrm{NH}_{2}\right), 3.82\left(\mathrm{~s}, 6 \mathrm{H}, \mathrm{OCH}_{3}\right)$; MS m/z : $252 \mathrm{M}^{+}$; Anal. Calcd. (\%) for $\mathrm{C}_{10} \mathrm{H}_{12} \mathrm{~N}_{4} \mathrm{O}_{2} \mathrm{~S}: \mathrm{C}, 47.61 ; \mathrm{H}, 4.79 ; \mathrm{N}, 22.21 ; \mathrm{S}, 12.71$. Found: C, 47.69; H, 4.81; N, 22.16; S, 12.73.

4-Amino-3- (3,5-dimethoxy phenyl) -5 - mercapto-1,2,4-triazole.(3b)

Yield:62\%; m.p: $200{ }^{\circ} \mathrm{C}$; IR (KBr) $\vee\left(\mathrm{cm}^{-1}\right): 3286$ (NH stretching), 1610 (C=N stretching); 3090 (aromatic CH stretching), 2580 (SH), 2934, 2847 (methyl CH stretch), 1264 (asymmetric C-O-C stretching), 1018 (symmetric C-O-C stretching), $1280(\mathrm{~N}-\mathrm{N}=\mathrm{C}), 1588,1548,1486,1455$ (C=C ring stretching); ${ }^{1} \mathrm{HNMR} \delta$ (ppm): 13.90 (s, 1H, SH), 7.2 (d, 2H, C-2 \& C-6 of Ar), 6.7 (d, 1H, C-4 of Ar), 5.84 (s, $2 \mathrm{H}, \mathrm{NH}_{2}$ ), 3.84 (s, $6 \mathrm{H}, \mathrm{OCH}_{3}$ ); $\mathrm{MS} \mathrm{m} / 2: 252 \mathrm{M}^{+}$; Anal. Calcd. (\%) for $\mathrm{C}_{10} \mathrm{H}_{12} \mathrm{~N}_{4} \mathrm{O}_{2} \mathrm{~S}$ : C, 47.61; H, 4.79; N, 22.21; S, 12.71. Found: C, 47.50; H, 4.84; N, 22.26; S, 12.68.

\section{4-Amino-3- (3,4,5-trimethoxy phenyl) -5 - mercapto -1,2,4-triazole. (3c)}

Yield: 60\%; m.p: $206{ }^{\circ} \mathrm{C}$; IR (KBr) $\vee\left(\mathrm{cm}^{-1}\right)$ : 3271 (NH stretching), 1607 (C=N stretching), 3092 (aromatic CH stretching), 1571, 1558, 1480, 1451 (C=C ring stretching), $2585(\mathrm{SH}), 2935,2838$ (methyl CH stretch), 1261 (asymmetric C-O-C stretching), 1037 (symmetric C-O-C stretching), $1287(\mathrm{~N}-\mathrm{N}=\mathrm{C}) ;{ }^{1} \mathrm{H} N M R \quad \delta(\mathrm{ppm}): 13.90$ (s, 1H, SH), 7.36 (s, 2H, C-2 \& C-6 of Ar), 5.82 (s, 2H, $\mathrm{NH}_{2}$ ), 3.76 (s, 9H, $\mathrm{OCH}_{3}$ ); MS m/z: $282 \mathrm{M}+$; Anal. Calcd. (\%) for $\mathrm{C}_{11} \mathrm{H}_{14} \mathrm{~N}_{4} \mathrm{O}_{3} \mathrm{~S}: \mathrm{C}, 46.80 ; \mathrm{H}$, 5.00; N, 19.85; S, 11.36. Found: C, 46.73; H, 4.97; N, 19.91; S, 11.33.

\section{4-Amino-3- (4-pyridinyl) -5 - mercapto -1,2,4-triazole. (3d)}

Yield: $65 \%$; m.p: $262{ }^{\circ} \mathrm{C}$; IR ( $\left.\mathrm{KBr}\right) \vee\left(\mathrm{cm}^{-1}\right)$ : 3271 (NH stretching), 1607 (C=N stretching), 3080 (aromatic $\mathrm{CH}$ stretching), 1571, 1558, 1480, 1451 (C=C ring stretching), $2585(\mathrm{SH}), 2935,2838$ (methyl CH stretch), 1261 (asymmetric C-O-C stretching), 1037 (symmetric C-O-C stretching), $1287(\mathrm{~N}-\mathrm{N}=\mathrm{C}) ;{ }^{1} \mathrm{H}$ NMR $\delta(\mathrm{ppm}): 14.10$ (s, 1H, SH), 8.0 (d, 2H, C-3 \& C-5 of Ar), 8.72 (d, 2H, C-2 \& C-6 of Ar), 5.84 (s, 2H, NH $)_{2}$; MS m/z: $193 \mathrm{M}^{+}$; Anal. Calcd. (\%) for $\mathrm{C}_{7} \mathrm{H}_{7} \mathrm{~N}_{5} \mathrm{~S}: \mathrm{C}, 43.51$; H, 3.65; N, 36.24; S, 16.59. Found: C, 43.42; H, 3.68; N, 36.17; S, 16.57 . 
General method for the synthesis of 3-aryl/heteroaryl- 6- (2-substituted-4quinolinyl)-1,2,4-triazolo [3,4-b]-1,3,4- thiadiazoles. 4(a-d)

A mixture of respective triazole $(0.02 \mathrm{~mol}), 2$-phenyl-quinoline-4-carboxylic acid $(0.02 \mathrm{~mol})$, and phosphorous oxychloride $(10 \mathrm{~mL})$ was heated under reflux for $4-6 \mathrm{~h}$. The reaction mixture was cooled to room temperature and the mixture was gradually poured onto crushed ice with stirring. Finely powdered potassium carbonate and the required amount of solid potassium hydroxide were added till the $\mathrm{pH}$ of the mixture was raised to 8 , to remove the excess of phosphorous oxychloride. Allowed the mixture to stand overnight, solid was separated. It was filtered, washed thoroughly with cold water, dried and recrystallized from hot ethanol. Similarly other compounds were prepared.

\section{3-(3,4-dimethoxyphenyl)-6-(2-phenyl-4-quinolinyl)-1,2,4-triazolo[3,4-b]-1,3,4-} thiadiazole (4a)

Yield: 52\%, m.p: $222-224{ }^{\circ} \mathrm{C}$; IR (KBr) $\vee\left(\mathrm{cm}^{-1}\right)$ : 3080 (aromatic $\mathrm{CH}$ stretching), $1604(\mathrm{C}=\mathrm{N}$ stretching), 1591, 1572, 1490, 1451 (C=C ring stretch), 2940, 2840 (methyl CH stretch), 1264 (asymmetric C-O-C stretching), 1014 (symmetric C-O-C stretching), 1280 (N-N=C); ${ }^{1} \mathrm{H}$ NMR $\delta$ (ppm): 7.14-8.2 (m, Ar-H), $3.80\left(\mathrm{~s}, 6 \mathrm{H}, \mathrm{OCH}_{3}\right)$; MS m/z: $465 \mathrm{M}^{+}$; Anal. Calcd. (\%) for $\mathrm{C}_{26} \mathrm{H}_{19} \mathrm{~N}_{5} \mathrm{O}_{2} \mathrm{~S}$ : C, 67.08; H, 4.11; N, 15.04; S, 6.89. Found: C, 67.19; H, 4.14; N, $15.10 ; \mathrm{S}, 6.86$.

\section{3-(3,5-dimethoxyphenyl)-6-(2-phenyl-4-quinolinyl)-1,2,4-triazolo[3,4-b]-1,3,4-} thiadiazole $(4 \mathrm{~b})$

Yield: $48 \%$, m.p: $232{ }^{\circ} \mathrm{C}$; IR (KBr) $\vee\left(\mathrm{cm}^{-1}\right)$ : 1611 (C=N stretching), 3085 (aromatic $\mathrm{CH}$ stretching), 1585, 1570, 1480 (C=C ring stretching), 2945, 2840 (methyl $\mathrm{CH}$ stretch), 1261 (asymmetric C-O-C stretching), 1021 (symmetric C-O-C stretching), $1280(\mathrm{~N}-\mathrm{N}=\mathrm{C}) ;{ }^{1} \mathrm{H}$ NMR $\delta$ (ppm): 6.7-8.2 (m, Ar-H), $3.82\left(\mathrm{~s}, 6 \mathrm{H}, \mathrm{OCH}_{3}\right) ; \mathrm{MS} \mathrm{m} / \mathrm{z}: 465$ $\mathrm{M}^{+}$; Anal. Calcd. (\%) for $\mathrm{C}_{26} \mathrm{H}_{19} \mathrm{~N}_{5} \mathrm{O}_{2} \mathrm{~S}$ : C, 67.08; H, 4.11; N, 15.04; S, 6.89. Found: C, 66.94; H, 4.08; N, 15.09; S, 6.85.

\section{3-(3, 4, 5-trimethoxy phenyl)-6- (2-phenyl-4-quinolinyl)-1,2,4-triazolo[3,4-b] - 1,3,4-thiadiazole (4c)}

Yield: $46 \%$, m.p: $186{ }^{\circ} \mathrm{C}$; IR $(\mathrm{KBr}) \vee\left(\mathrm{cm}^{-1}\right)$ : 3095 (aromatic $\mathrm{CH}$ stretching), $1614(\mathrm{C}=\mathrm{N}$ stretching), 1585, 1551, 1479, 1453 (C=C ring stretch), 1254 (asymmetric C-O-C stretching), 1024 (symmetric C-O-C stretching), 2955, 2840 (methyl CH stretch), 1274 (N$\mathrm{N}=\mathrm{C}) ;{ }^{1} \mathrm{H}$ NMR $\delta(\mathrm{ppm}):$ 7.3-8.2 (m, 12H, Ar-H), $3.86\left(\mathrm{~s}, 9 \mathrm{H}, \mathrm{OCH}_{3}\right) ; \mathrm{MS} \mathrm{m} / \mathrm{z}: 495 \mathrm{M}^{+}$; Anal. Calcd. (\%) for $\mathrm{C}_{27} \mathrm{H}_{21} \mathrm{~N}_{5} \mathrm{O}_{3} \mathrm{~S}$ : C, 65.44; H, 4.27; N, 14.13; S, 6.47. Found: C, 65.57; $\mathrm{H}, 4.24 ; \mathrm{N}, 14.07 ; \mathrm{S}, 6.43$.

3-(4-pyridinyl)-6- (2-phenyl-4-quinolinyl)-1,2,4-triazolo [3,4-b] -1,3,4thiadiazole $(4 d)$

Yield: 54\%, m.p: $212^{\circ} \mathrm{C}$; IR $(\mathrm{KBr}) \vee\left(\mathrm{cm}^{-1}\right)$ : 3080 (aromatic $\mathrm{CH}$ stretching), $1611(\mathrm{C}=\mathrm{N}$ stretching), 1580, $1560\left(\mathrm{C}=\mathrm{C}\right.$ ring stretch), $1286(\mathrm{~N}-\mathrm{N}=\mathrm{C}) ;{ }^{1} \mathrm{H}$ NMR $\delta(\mathrm{ppm}):$ 7.4-8.2 (m, $13 \mathrm{H}, \mathrm{Ar}-\mathrm{H}), 8.70$ (d, 2H, C-3 \& C-5 of Ar); MS m/z: $406 \mathrm{M}^{+}$; Anal. Calcd. (\%) for $\mathrm{C}_{23} \mathrm{H}_{14}$ $\mathrm{N}_{6}$ S: C, 67.96; H, 3.47; N, 20.68; S, 7.89. Found: C, 68.07; H, 3.45; N, 20.79; S, 7.85. 
3-(3,4-dimethoxyphenyl)-6-(2-methyl-4-quinolinyl)-1,2,4-triazolo[3,4-b]-1,3,4-thiadiazole (5a)

Yield: 56\%; m.p: $170^{\circ} \mathrm{C}$; IR (KBr) $\vee\left(\mathrm{cm}^{-1}\right)$ : 3085 (aromatic $\mathrm{CH}$ stretching), 1610 (C=N stretching), 1594, 1480, 1450 ( $\mathrm{C}=\mathrm{C}$ ring stretch), 2960, 2840 (methyl $\mathrm{CH}$ stretch), 1260 (asymmetric C-O-C

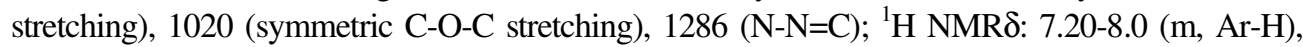
$3.80\left(\mathrm{~s}, 3 \mathrm{H}, \mathrm{OCH}_{3}\right), 2.78\left(\mathrm{~s}, 3 \mathrm{H}, \mathrm{CH}_{3}\right)$; $\mathrm{MS} m / z: 404 \mathrm{M}^{+}$; Anal. Calcd. (\%) for $\mathrm{C}_{21} \mathrm{H}_{17} \mathrm{~N}_{5} \mathrm{O}_{2} \mathrm{~S}: \mathrm{C}$, 62.52; H, 4.25; N, 17.36; S, 7.95. Found: C, 62.61; H, 4.25; N, 17.39; S, 7.92.

3-(3,5-dimethoxyphenyl)-6-(2-methyl-4-quinolinyl)-1,2,4-triazolo[3,4-b]-1,3,4-thiadiazole (5b)

Yield: 52\%; m.p: $186^{\circ} \mathrm{C}$; IR (KBr) v $\left(\mathrm{cm}^{-1}\right)$ : 1608 (C=N stretching), 3080 (aromatic $\mathrm{CH}$ stretching), 1580, 1560, 1480(C=C ring stretching), 2942, 2846 (methyl $\mathrm{CH}$ stretch), 1266 (asymmetric C-O-C stretching), 1024 (symmetric C-O-C stretching), $1280(\mathrm{~N}-\mathrm{N}=\mathrm{C}) ;{ }^{1} \mathrm{H}$ NMR $\delta$ (ppm): 6.72-8.02 (m, Ar-H), $3.84\left(\mathrm{~s}, 6 \mathrm{H}, \mathrm{OCH}_{3}\right), 2.80\left(\mathrm{~s}, 3 \mathrm{H}, \mathrm{CH}_{3}\right) ; \mathrm{MS} \mathrm{m} / z$ : $403 \mathrm{M}^{+}$; Anal. Calcd. (\%) for $\mathrm{C}_{21} \mathrm{H}_{17} \mathrm{~N}_{5} \mathrm{O}_{2}$ S: C, 62.52; H, 4.25; N, 17.36; S, 7.95. Found: C, 62.44; H, 4.21; N, 17.32; S, 7.98.

\section{3-(3, 4, 5-trimethoxy phenyl)-6- (2-methyl-4-quinolinyl)-1,2,4-triazolo [3,4-b] - 1,3,4-thiadiazole $(5 \mathrm{c})$}

Yield: 53\%; m.p: $178^{\circ} \mathrm{C}$; IR $(\mathrm{KBr}) \vee\left(\mathrm{cm}^{-1}\right)$ : 3090 (aromatic $\mathrm{CH}$ stretching), $1610(\mathrm{C}=\mathrm{N}$ stretching), 1580, 1561, 1484 ( $\mathrm{C}=\mathrm{C}$ ring stretch), 2965, 2848 (methyl $\mathrm{CH}$ stretch), 1262 (asymmetric C-O-C stretching), 1020 (symmetric C-O-C stretching), $1276(\mathrm{~N}-\mathrm{N}=\mathrm{C}) ;{ }^{1} \mathrm{H}$ NMR $\delta$ (ppm): 7.30-8.02 (m, Ar-H), $3.86\left(\mathrm{~s}, 9 \mathrm{H}, \mathrm{OCH}_{3}\right), 2.82\left(\mathrm{~s}, 3 \mathrm{H}, \mathrm{CH}_{3}\right.$ of $\left.\mathrm{Ar}^{\prime}\right) ; \mathrm{MS} \mathrm{m} / z$ : $433 \mathrm{M}^{+}$; Anal. Calcd. (\%) for $\mathrm{C}_{22} \mathrm{H}_{19} \mathrm{~N}_{5} \mathrm{O}_{3} \mathrm{~S}$ : C, 60.96; H, 4.42; N, 16.16; S, 7.40. Found: C, 61.09; H, 4.39; N, 16.21; S, 7.38.

\section{3-(4-pyridinyl)-6- (2-methyl-4-quinolinyl)-1,2,4-triazolo [3,4-b] -1,3,4-thiadiazole (5d)}

Yield: 45\%; m.p: $206^{\circ} \mathrm{C}$; IR $(\mathrm{KBr}) \vee\left(\mathrm{cm}^{-1}\right)$ : 3080 (aromatic $\mathrm{CH}$ stretching), $1611(\mathrm{C}=\mathrm{N}$ stretching), 1580, $1560\left(\mathrm{C}=\mathrm{C}\right.$ ring stretch), $1286(\mathrm{~N}-\mathrm{N}=\mathrm{C}) ;{ }^{1} \mathrm{H}$ NMR $\delta: 7.32-8.1(\mathrm{~m}, 5 \mathrm{H}$ of Ar'), 8.42 (d, 2H, C-3 \& C-5 of Ar); 8.80 (d, 2H, C-2 \& C-6 of Ar); 2.8 (s, 3H, $\mathrm{CH}_{3}$ ); MS $m / z$ : $344 \mathrm{M}^{+}$; Anal. Calcd. (\%) for $\mathrm{C}_{18} \mathrm{H}_{12} \mathrm{~N}_{6} \mathrm{~S}: \mathrm{C}, 62.77 ; \mathrm{H}, 3.51 ; \mathrm{N}, 24.40 ; \mathrm{S}, 9.31$. Found: C, 62.85; H, 3.49; N, 24.47; S, 9.28.

\section{3-(3,4-dimethoxy phenyl)-6-(4-quinolinyl)-1,2,4-triazolo[3,4-b]-1,3,4-thiadiazole (6a)}

Yield: $46 \%$; m.p: $240{ }^{\circ} \mathrm{C}$; IR (KBr) v $\left(\mathrm{cm}^{-1}\right)$ : 3073 (aromatic CH stretching), 1607 (C=N stretching), 1592, 1570 ( $\mathrm{C}=\mathrm{C}$ ring stretch), 2945, 2854 (methyl $\mathrm{CH}$ stretch), 1267 (asymmetric C-O-C stretching), 1023 (symmetric C-O-C stretching), 1275 (N-N=C); ${ }^{1} \mathrm{H}$ NMR $\delta$ (ppm): 7.18-7.7 (m, Ar-H), 8.05 (d, 1H, C-8' of Ar'), 8.88 (d, 1H, C-2' of Ar'), 3.80 (s, 6H, $\left.\mathrm{OCH}_{3}\right)$; MS m/z: 389 M'; Anal. Calcd. (\%) for $\mathrm{C}_{20} \mathrm{H}_{15} \mathrm{~N}_{5} \mathrm{O}_{2} \mathrm{~S}: \mathrm{C}, 61.68 ; \mathrm{H}, 3.88 ; \mathrm{N}, 17.98 ; \mathrm{S}, 8.23$. Found: C, 61.56; H, 3.91; N, 18.05; S, 8.19.

\section{3-(3,5-dimethoxy phenyl)-6-(4-quinolinyl)-1,2,4-triazolo [3,4-b]-1,3,4-thiadiazole (6b)}

Yield: $49 \%$; m. p: $248^{\circ} \mathrm{C}$; IR $(\mathrm{KBr}) \vee\left(\mathrm{cm}^{-1}\right): 3065$ (aromatic $\mathrm{CH}$ stretching), 1586, 1566 ( $\mathrm{C}=\mathrm{C}$ ring stretching), 2960, 2854 (methyl $\mathrm{CH}$ stretch), $1616(\mathrm{C}=\mathrm{N}$ stretching), 1258 (asymmetric C-O-C stretching), 1020 (symmetric C-O-C stretching), $1281(\mathrm{~N}-\mathrm{N}=\mathrm{C}) ;{ }^{1} \mathrm{H}$ NMR $\delta$ (ppm): 6.70-7.7 (m, Ar-H), 8.05 (d, 1H, C-8' of Ar'), 8.9 (d, 1H, C-2' of Ar'), 3.84 (s, $6 \mathrm{H}, \mathrm{OCH}_{3}$ ); MS m/z: $389 \mathrm{M}^{+}$; Anal. Calcd. (\%) for $\mathrm{C}_{20} \mathrm{H}_{15} \mathrm{~N}_{5} \mathrm{O}_{2} \mathrm{~S}: \mathrm{C}, 61.68 ; \mathrm{H}, 3.88 ; \mathrm{N}$, 17.98; S, 8.23. Found: C, 61.79; H, 3.85; N, 18.05; S, 8.20. 
3-(3, 4, 5-trimethoxy phenyl)-6- (4-quinolinyl)-1,2,4-triazolo [3,4-b] -1,3,4thiadiazole (6c)

Yield: 47\%; m.p: $234^{\circ} \mathrm{C}$; IR (KBr) $\vee\left(\mathrm{cm}^{-1}\right)$ : 3078 (aromatic $\mathrm{CH}$ stretching), $1614(\mathrm{C}=\mathrm{N}$ stretching), 1590, 1575 ( $\mathrm{C}=\mathrm{C}$ ring stretch), 2970, 2848 (methyl $\mathrm{CH}$ stretch), 1263 (asymmetric C-O-C stretching), 1018 (symmetric C-O-C stretching), $1284(\mathrm{~N}-\mathrm{N}=\mathrm{C}) ;{ }^{1} \mathrm{H}$ NMR $\delta(\mathrm{ppm}):$ 7.30-7.74 (m, Ar-H), 8.05 (d, 1H, C-8' of Ar'), 8.9 (d, 1H, C-2' of Ar'), $3.86\left(\mathrm{~s}, 9 \mathrm{H}, \mathrm{OCH}_{3}\right)$; MS m/z: $419 \mathrm{M}^{+}$; Anal. Calcd. (\%) for $\mathrm{C}_{21} \mathrm{H}_{17} \mathrm{~N}_{5} \mathrm{O}_{3} \mathrm{~S}: \mathrm{C}, 60.13 ; \mathrm{H}$, 4.09; N, 16.70; S, 7.64. Found: C, 60.01; H, 4.12; N, 16.65; S, 7.61.

\section{3-(4-pyridinyl)-6- (4-quinolinyl)-1,2,4-triazolo[3,4-b] -1,3,4-thiadiazole (6d)}

Yield: 54\%; m.p: $284^{\circ} \mathrm{C}$; IR ( $\left.\mathrm{KBr}\right) \vee\left(\mathrm{cm}^{-1}\right)$ : 3080 (aromatic $\mathrm{CH}$ stretching), 1611 $(\mathrm{C}=\mathrm{N}$ stretching $), 1580,1560(\mathrm{C}=\mathrm{C}$ ring stretch $), 1286(\mathrm{~N}-\mathrm{N}=\mathrm{C}) ;{ }^{1} \mathrm{H}$ NMR $\delta: ~ 7.4-7.7$ (m, 4H, of Ar'), 7.96 (d, 2H, C-2 \& C-6 of Ar), 8.7 (d, 2H, C-2 \& C-6 of Ar), 8.05 (d, $1 \mathrm{H}, \mathrm{C}-8$ ' of Ar'), 8.9 (d, 1H, C-2' of Ar'); MS m/z: $330 \mathrm{M}^{+}$; Anal. Calcd. (\%) for $\mathrm{C}_{17} \mathrm{H}_{10} \mathrm{~N}_{6} \mathrm{~S}: \mathrm{C}, 61.80 ; \mathrm{H}, 3.05 ; \mathrm{N}, 25.44 ; \mathrm{S}, 9.71$. Found: C, 61.73; H, 3.07; N, $25.40 ; \mathrm{S}, 9.68$.

3-(3, 4-dimethoxy phenyl)-6- (2, 6-dihydroxy-4-pyridinyl)-1, 2, 4-triazolo [3,4-b]-1, 3, 4-thiadiazole (7a)

Yield: 50\%; m.p: $280^{\circ} \mathrm{C}\left(\mathrm{dec}\right.$ )); IR (KBr) $v\left(\mathrm{~cm}^{-1}\right)$ : 3430 (OH stretching), 3074 (aromatic CH stretching), 1612 ( $\mathrm{C}=\mathrm{N}$ stretching), 1590, 1541, 1480, 1455 ( $\mathrm{C}=\mathrm{C}$ ring stretch), 2965, 2931 (methyl CH stretch), 1260 (asymmetric C-O-C stretching), 1026 (symmetric C-O-C stretching), $1290(\mathrm{~N}-\mathrm{N}=\mathrm{C}) ;{ }^{1} \mathrm{H}$ NMR $\delta$ (ppm): 7.12 (d, 1H, C-5 of Ar), 7.52 (s, 1H, C-2 of Ar), 7.66 (d, 1H, C-6 of Ar), 7.26 (s, 2H, C-3 \& C-5 of Ar'), 6.14 (s, 2H, OH), 3.8 (s, 6H, $\mathrm{OCH}_{3}$ ); MS $m / z$ : $371 \mathrm{M}^{+}$; Anal. Calcd. (\%) for $\mathrm{C}_{16} \mathrm{H}_{13} \mathrm{~N}_{5} \mathrm{O}_{4} \mathrm{~S}: \mathrm{C}, 51.75 ; \mathrm{H}, 3.53 ; \mathrm{N}, 18.86$; S, 8.63. Found: C, 51.64; H, 3.56; N, 18.81; S, 8.59.

\section{3-(3, 5-dimethoxy phenyl)-6- (2,6-dihydroxy-4-pyridinyl)-1,2,4-triazolo[3,4-b]- 1,3,4- thiadiazole $(7 b)$}

Yield: 52\%; m.p: $294{ }^{\circ} \mathrm{C}$ (dec.); IR (KBr) $v\left(\mathrm{~cm}^{-1}\right)$ : 3443 (OH stretching), 3076, 3034 (aromatic $\mathrm{CH}$ stretching), 1607(C=N stretching), 1590, 1538, 1478, 1448 (C=C ring stretch), 2970, 2934 (methyl CH stretch), 1262 (asymmetric C-O-C stretching), 1015 (symmetric CO-C stretching), $1283(\mathrm{~N}-\mathrm{N}=\mathrm{C}) ;{ }^{1} \mathrm{H}$ NMR $\delta$ (ppm): 6.74 (d, 1H, C-4 of Ar), 7.20 (d, 2H, C-2 \& C-6 of Ar), 7.28 (s, 2H, C-3 \& C-5 of Ar'), 6.20 (s, 2H, OH), $3.84\left(\mathrm{~s}, 6 \mathrm{H}, \mathrm{OCH}_{3}\right)$; MS $m / z$ : $371 \mathrm{M}^{+}$; Anal. Calcd. (\%) for $\mathrm{C}_{16} \mathrm{H}_{13} \mathrm{~N}_{5} \mathrm{O}_{4} \mathrm{~S}: \mathrm{C}, 51.75 ; \mathrm{H}, 3.53 ; \mathrm{N}, 18.86 ; \mathrm{S}, 8.63$. Found: C, 51.67; H, 3.53; N, 18.91; S, 8.62.

\section{3-(3, 4, 5-trimethoxy phenyl)-6-(2, 6-dihydroxy-4-pyridinyl)-1, 2, 4-triazolo[3,4-b]- 1, 3,4-thiadiazole (7c)}

Yield: 50\%; m.p: $282{ }^{\circ} \mathrm{C}$ (dec.); IR (KBr) $\vee\left(\mathrm{cm}^{-1}\right): 3450(\mathrm{OH}$ stretching), 3080 (aromatic $\mathrm{CH}$ stretching), $1610(\mathrm{C}=\mathrm{N}$ stretching), 1590, 1564, 1482, 1448 ( $\mathrm{C}=\mathrm{C}$ ring stretch), 2958, 2930 (methyl $\mathrm{CH}$ stretch), 1264 (asymmetric C-O-C stretching), 1023 (symmetric C-O-C stretching), $1286(\mathrm{~N}-\mathrm{N}=\mathrm{C}) ;{ }^{1} \mathrm{H}$ NMR $\delta(\mathrm{ppm}): 7.36$ (s, 2H, C-2 \& C-6 of Ar), 7.24 (s, 2H, C-3 \& C-5 of Ar'), $3.88\left(\mathrm{~s}, 9 \mathrm{H}, \mathrm{OCH}_{3}\right), 6.20(\mathrm{~s}, 2 \mathrm{H}$, $\mathrm{OH}$ ); MS m/z: $401 \mathrm{M}^{+}$; Calcd. (\%) for $\mathrm{C}_{17} \mathrm{H}_{15} \mathrm{~N}_{5} \mathrm{O}_{5} \mathrm{~S}: \mathrm{C}, 50.87 ; \mathrm{H}, 3.77 ; \mathrm{N}, 17.45$; S, 7.99. Found: C, 50.81; H, 3.74; N, 17.51; S, 7.95. 
3-(4-pyridinyl)-6-(2, 6-dihydroxy-4-pyridinyl)-1,2,4-triazolo[3,4-b]-1,3,4thiadiazole $(7 d)$

Yield: 48\%; m.p: $300{ }^{\circ} \mathrm{C}$ (dec.); IR (KBr) $\vee\left(\mathrm{cm}^{-1}\right)$ : 3442 (OH stretching), 3087, (aromatic $\mathrm{CH}$ stretching), $1612(\mathrm{C}=\mathrm{N}$ stretching), 1585, $1558(\mathrm{C}=\mathrm{C}$ ring stretch), 1283 $(\mathrm{N}-\mathrm{N}=\mathrm{C}) ;{ }^{1} \mathrm{H}$ NMR $\delta(\mathrm{ppm}): 8.00(\mathrm{~d}, 2 \mathrm{H}, \mathrm{C}-3 \& \mathrm{C}-5$ of Ar), $8.72(\mathrm{~d}, 2 \mathrm{H}, \mathrm{C}-2 \&$ C-6 of Ar), 7.24 (s, 2H, C-3' \& C-5' of Ar'), 6.20 (s, 2H, OH); MS m/z: $312 \mathrm{M}^{+}$; Anal. Calcd. (\%) for $\mathrm{C}_{13} \mathrm{H}_{8} \mathrm{~N}_{6} \mathrm{O}_{2} \mathrm{~S}$ : C, 50.00; H, 2.58; N, 26.91; S, 10.27. Found: C, 49.91; $\mathrm{H}, 2.60 ; \mathrm{N}, 26.83 ; \mathrm{S}, 10.25$.

3-(3, 4-dimethoxy phenyl)-6- (2-chloro-4-pyridinyl)-1, 2, 4-triazolo [3,4-b]-1, 3, 4thiadiazole (8a)

Yield: $50 \%$; m.p: $218^{\circ} \mathrm{C}$; IR $(\mathrm{KBr}) \vee\left(\mathrm{cm}^{-1}\right): 3065$ (aromatic $\mathrm{CH}$ stretching), $1610(\mathrm{C}=\mathrm{N}$ stretching), 1585, 1567, 1484, 1455 (C=C ring stretch), 2970, 2840 (methyl $\mathrm{CH}$ stretch), 1254 (asymmetric C-O-C stretching), 1034(symmetric C-O-C stretching), 1275 (N-N=C); ${ }^{1} \mathrm{H}$ NMR $\delta(\mathrm{ppm}): 7.16$ (d, 1H, C-5 of Ar), 7.54 (s, 1H, C-2 of Ar), 7.68 (d, 1H, C-6 of Ar), 8.00 (s, 1H, C-3' of Ar'), 7.9 (d, 1H, C-5' of Ar'), 8.9 (d, 1H, C-6' of Ar'), 3.82 (s, 6H, $\mathrm{OCH}_{3}$ ); MS m/z: $373 \mathrm{M}^{+}, 375 \mathrm{M}^{+2}$; Anal. Calcd. (\%) for $\mathrm{C}_{16} \mathrm{H}_{12} \mathrm{~N}_{5} \mathrm{O}_{2} \mathrm{~S} \mathrm{Cl}: \mathrm{C}, 51.41 ; \mathrm{H}$, 3.24; N, 18.73; S, 8.58. Found: C, 51.52; H, 3.27; N, 18.64; S, 8.54.

3-(3,5-dimethoxy phenyl)-6- (2-chloro-4-pyridinyl)-1,2,4-triazolo [3,4-b]-1,3,4thiadiazole $(8 b)$

Yield: 47\%; m.p: $224{ }^{\circ} \mathrm{C}$; IR (KBr) v $\left(\mathrm{cm}^{-1}\right)$ : 3084, 3048 (aromatic $\mathrm{CH}$ stretching), 1607 (C=N stretching), 1585, 1554, 1464, 1448 (C=C ring stretch), 2984, 2854 (methyl $\mathrm{CH}$ stretch), 1260 (asymmetric C-O-C stretching), 1028 (symmetric C-O-C stretching), 1284 (N$\mathrm{N}=\mathrm{C}$ ); ${ }^{1} \mathrm{H}$ NMR $\delta$ (ppm): 7.26 (d, 2H, C-2 \& C-6 of Ar), 6.74 (d, 1H, C-4 of Ar), 8.00 (s, 1H, C-3' of Ar'), 7.9 (d, 1H, C-5' of Ar'), 8.88 (d, 1H, C-6' of Ar'), 3.86 (s, 6H, OCH 3 ); MS $m / z: 373 \mathrm{M}^{+}, 375 \mathrm{M}^{+2}$; Anal. Calcd. (\%) for $\mathrm{C}_{16} \mathrm{H}_{12} \mathrm{~N}_{5} \mathrm{O}_{2} \mathrm{~S} \mathrm{Cl}: \mathrm{C}, 51.41 ; \mathrm{H}, 3.24 ; \mathrm{N}, 18.73$; S, 8.58. Found: C, 51.37; H, 3.24; N, 18.69; S, 8.56.

3-(3, 4, 5-trimethoxy phenyl)-6-(2-chloro-4-pyridinyl)-1, 2, 4-triazolo[3,4-b]-1, 3,4thiadiazole $(8 c)$

Yield: $48 \%$; m.p: $226^{\circ} \mathrm{C}(\mathrm{dec}$ ) $)$ IR $(\mathrm{KBr}) \vee\left(\mathrm{cm}^{-1}\right)$ : 3080 (aromatic $\mathrm{CH}$ stretching), 1614 (C=N stretching), 1587, 1550, $1482 \quad(\mathrm{C}=\mathrm{C}$ ring stretch), 1264 (asymmetric C-O-C stretching), 1017 (symmetric C-O-C stretching), 2958, 2854 (methyl CH stretch), 1276 (N$\mathrm{N}=\mathrm{C}$ ); ${ }^{1} \mathrm{H}$ NMR $\delta$ (ppm): 7.34 (s, 2H, C-2 \& C-6 of Ar), 8.00 (s, 1H, C-3' of Ar'), 7.9 (d, $1 \mathrm{H}, \mathrm{C}-5$ ' of Ar'), 8.90 (d, 1H, C-6' of Ar'), 3.86 (s, 9H, $\mathrm{OCH}_{3}$ ); MS m/z: $403 \mathrm{M}^{+}, 405 \mathrm{M}^{+2}$; Anal. Calcd. (\%) for $\mathrm{C}_{17} \mathrm{H}_{14} \mathrm{~N}_{5} \mathrm{O}_{3} \mathrm{~S} \mathrm{Cl}$ : C, 50.56; H, 3.49; N, 17.34; S, 7.94. Found: C, 50.47; H, 3.50; N, 17.29; S, 7.91.

\section{3-(4-pyridinyl)-6-(2-chloro-4-pyridinyl)-1,2,4-triazolo[3,4-b]-1,3,4-thiadiazole (8d)}

Yield: $48 \%$; m.p: $270^{\circ} \mathrm{C}\left(\mathrm{dec}\right.$ ); IR $(\mathrm{KBr}) \vee\left(\mathrm{cm}^{-1}\right)$ : 3080 (aromatic $\mathrm{CH}$ stretching), $1611\left(\mathrm{C}=\mathrm{N}\right.$ stretching), 1580, $1560(\mathrm{C}=\mathrm{C}$ ring stretch $), 1286(\mathrm{~N}-\mathrm{N}=\mathrm{C}) ;{ }^{1} \mathrm{H}$ NMR $\delta$ (ppm): 8.00 (d, 2H, C-3 \& C-5 of Ar), 8.7 (d, 2H, C-2 \& C-6 of Ar), 8.02 (s, 1H, C-3' of Ar'), 7.9 (d, 1H, C-5' of Ar'), 8.90 (d, 1H, C-6' of Ar'); MS m/z: $314 \mathrm{M}^{+}, 316 \mathrm{M}^{+2}$; Anal. Calcd. (\%) for $\mathrm{C}_{13} \mathrm{H}_{7} \mathrm{~N}_{6} \mathrm{~S} \mathrm{Cl}$ : C, 49.61; H, 2.24; N, 26.70; S, 10.19. Found: C, 49.52; H, 2.26; N, 26.75; S, 10.14 . 
3-(3, 4-dimethoxy phenyl)-6- (2-flouro-4-pyridinyl)-1, 2, 4-triazolo [3,4-b]-1, 3, 4thiadiazole (9a)

Yield: $49 \%$; m.p: $206^{\circ} \mathrm{C}\left(\mathrm{dec}\right.$ )); IR $(\mathrm{KBr}) \vee\left(\mathrm{cm}^{-1}\right)$ : 3082 (aromatic $\mathrm{CH}$ stretching), 1618 $(\mathrm{C}=\mathrm{N}$ stretching), 1580, 1564 ( $\mathrm{C}=\mathrm{C}$ ring stretch), 2964, 2846 (methyl $\mathrm{CH}$ stretch), 1258 (asymmetric C-O-C stretching), 1028(symmetric C-O-C stretching), $1280(\mathrm{~N}-\mathrm{N}=\mathrm{C}) ;{ }^{1} \mathrm{H}$ NMR $\delta$ (ppm): 7.12 (d, 1H, C-5 of Ar), 7.56 (s, 1H, C-2 of Ar), 7.76 (d, 1H, C-6 of Ar), 7.7 (s, 1H, C-3' of Ar'), 7.9 (d, 1H, C-5' of Ar'), 8.60 (d, 1H, C-6' of Ar'), 3.86 (s, 6H, $\mathrm{OCH}_{3}$ ); MS m/z: $357 \mathrm{M}^{+}$; Anal. Calcd. (\%) for $\mathrm{C}_{16} \mathrm{H}_{12} \mathrm{~N}_{5} \mathrm{O}_{2} \mathrm{~S}$ F: C, 53.77; H, 3.38; N, 19.60; S, 8.97. Found: C, 53.68; H, 3.41; N, 19.64; S, 8.93.

\section{3-(3,5-dimethoxyphenyl)-6-(2-flouro-4-pyridinyl)-1,2,4-triazolo[3,4-b]-1,3,4-thiadiazole(9b)}

Yield: $46 \%$; m.p: $190^{\circ} \mathrm{C}(\mathrm{dec})$; IR $(\mathrm{KBr}) \vee\left(\mathrm{cm}^{-1}\right)$ : 3090 (aromatic $\mathrm{CH}$ stretching), 1617 $(\mathrm{C}=\mathrm{N}$ stretching), 1575, 1560 ( $\mathrm{C}=\mathrm{C}$ ring stretch), 2954, 2837 (methyl $\mathrm{CH}$ stretch), 1260 (asymmetric C-O-C stretching), 1024 (symmetric C-O-C stretching), $1284(\mathrm{~N}-\mathrm{N}=\mathrm{C}) ;{ }^{1} \mathrm{H}$ NMR $\delta$ (ppm): 7.24 (d, 2H, C-2 \& C-6 of Ar), 6.72 (d, 1H, C-4 of Ar), 7.68 (s, 1H, C-3' of Ar'), 7.90(d, 1H, C-5' of Ar'), 8.60 (d, 1H, C-6' of Ar'), 3.80 (s, 6H, $\mathrm{OCH}_{3}$ ); MS m/z: 357 $\mathrm{M}^{+}$; Anal. Calcd. (\%) for $\mathrm{C}_{16} \mathrm{H}_{12} \mathrm{~N}_{5} \mathrm{O}_{2} \mathrm{~S}$ F: C, 53.77; H, 3.38; N, 19.60; S, 8.97. Found: C, 53.83; H, 3.36; N, 19.55; S, 8.95.

3-(3, 4, 5-trimethoxy phenyl)-6-(2-flouro-4-pyridinyl)-1, 2, 4-triazolo[3,4-b]-1, 3,4thiadiazole $(9 c)$

Yield: $53 \%$; m.p: $210^{\circ} \mathrm{C}(\mathrm{dec})$; IR $(\mathrm{KBr}) \vee\left(\mathrm{cm}^{-1}\right)$ : 3080 (aromatic $\mathrm{CH}$ stretching), 1614 (C=N stretching), 1587, 1550, $1482 \quad(\mathrm{C}=\mathrm{C}$ ring stretch), 1267 (asymmetric C-O-C stretching), 1019 (symmetric C-O-C stretching), 2958, 2854 (methyl CH stretch), 1276 (N$\mathrm{N}=\mathrm{C}$ ); ${ }^{1} \mathrm{H}$ NMR $\delta$ (ppm): ${ }^{1} \mathrm{H}$ NMR $\delta$ (ppm): 7.32 (s, 2H of Ar), 7.68 (s, 1H, C-3' of Ar'), 7.88 (d, 1H, C-5' of Ar'), 8.58 (d, 1H, C-6' of Ar'), 3.86 (s, 9H, $\mathrm{OCH}_{3}$ ); $\mathrm{MS} \mathrm{m} / z: 387 \mathrm{M}^{+}$; Anal. Calcd. (\%) for $\mathrm{C}_{17} \mathrm{H}_{14} \mathrm{~N}_{5} \mathrm{O}_{3}$ S F: C, 52.71; H, 3.64; N, 18.08; S, 8.28. Found: C, 52.65; H, 3.64; N, 18.15; S, 8.32.

\section{3-(4-pyridinyl)-6-(2-flouro-4-pyridinyl)-1,2,4-triazolo[3,4-b]-1,3,4-thiadiazole (9d)}

Yield: $51 \%$; m.p: $206^{\circ} \mathrm{C}(\mathrm{dec})$; IR $(\mathrm{KBr}) \vee\left(\mathrm{cm}^{-1}\right): 3080$ (aromatic $\mathrm{CH}$ stretching), 1611 $\left(\mathrm{C}=\mathrm{N}\right.$ stretching), 1580, $1560\left(\mathrm{C}=\mathrm{C}\right.$ ring stretch), $1286(\mathrm{~N}-\mathrm{N}=\mathrm{C}) ;{ }^{1} \mathrm{H}$ NMR $\delta(\mathrm{ppm}): 8.0(\mathrm{~d}$, 2H, C-3 \& C-5 of Ar), 8.8 (d, 2H, C-2 \& C-6 of Ar), 7.68 (s, 1H, C-3' of Ar'), 7.88 (d, 1H, C-5' of Ar'), 8.62 (d, 1H, C-6' of Ar'); MS m/z: $298 \mathrm{M}^{+}$; Anal. Calcd. (\%) for $\mathrm{C}_{13} \mathrm{H}_{7} \mathrm{~N}_{6} \mathrm{~S}$ F: C, 52.34; H, 2.37; N, 28.17; S, 10.75. Found: C, 52.25; H, 2.39; N, 28.24; S, 10.71.

3-(3, 4-dimethoxy phenyl)-6-(5-methoxy-3-indolyl methyl)-1,2,4-triazolo[3,4-b]1,3,4-thiadiazole (10a)

Yield: $48 \%$; m.p: $160^{\circ} \mathrm{C}$; IR $(\mathrm{KBr}) \vee\left(\mathrm{cm}^{-1}\right)$ : 3084 (aromatic $\mathrm{CH}$ stretching), $1614(\mathrm{C}=\mathrm{N}$ stretching), 1587, 1565 ( $\mathrm{C}=\mathrm{C}$ ring stretch), 2980, 2849 (methyl $\mathrm{CH}$ stretch), 1265 (asymmetric C-O-C stretching), 1018 (symmetric C-O-C stretching), $1282(\mathrm{~N}-\mathrm{N}=\mathrm{C}) ;{ }^{1} \mathrm{H}$ NMR $\delta(\mathrm{ppm}): 7.16(\mathrm{~d}, 1 \mathrm{H}$ of $\mathrm{Ar}), 7.52(\mathrm{~s}, 1 \mathrm{H}, \mathrm{C}-2 \mathrm{of} \mathrm{Ar}), 7.68(\mathrm{~d}, 1 \mathrm{H}, \mathrm{C}-6$ of Ar), $7.4(\mathrm{~m}$, 2H, C-4' \& C-6' of Ar'), 7.78 (d, 1H, C-7' of Ar'), 6.86 (s, 1H, C-2' of Ar'), 3.82 (s, 6H, $\mathrm{OCH}_{3}$ in $\mathrm{Ar}$ ), 3.74 (s, $3 \mathrm{H}, \mathrm{OCH}_{3}$ in $\left.\mathrm{Ar}^{\prime}\right), 4.10\left(\mathrm{~s}, 2 \mathrm{H}, \mathrm{CH}_{2}\right), 10.10$ (s, 1H, NH of Ar); MS $m / z: 421 \mathrm{M}^{+}$; Anal. Calcd. (\%) for $\mathrm{C}_{21} \mathrm{H}_{19} \mathrm{~N}_{5} \mathrm{O}_{3} \mathrm{~S}: \mathrm{C}, 59.84 ; \mathrm{H}, 4.54 ; \mathrm{N}, 16.62 ; \mathrm{S}, 7.61$. Found: C, 59.75; H, 4.54; N, 16.67; S, 7.58. 
3-(3, 5-dimethoxy phenyl)-6-(5-methoxy-3-indolyl methyl)-1,2,4-triazolo[3,4-b]1,3,4-thiadiazole (10b)

Yield: $50 \%$; m.p: $176^{\circ} \mathrm{C}$; IR $(\mathrm{KBr}) \vee\left(\mathrm{cm}^{-1}\right)$ : 3070 (aromatic $\mathrm{CH}$ stretching), $1624(\mathrm{C}=\mathrm{N}$ stretching), 1588, 1546 ( $\mathrm{C}=\mathrm{C}$ ring stretch), 2956, 2864 (methyl $\mathrm{CH}$ stretch), 1260 (asymmetric CO-C stretching), 1021 (symmetric C-O-C stretching), 1285 (N-N=C); ${ }^{1} \mathrm{H}$ NMR $\delta$ (ppm): 7.20 (d, 2H, C-2 \& C-6 of Ar), 6.76 (d, 1H, C-4 of Ar), 7.40 (m, 2H, C-4' \& C-6' of Ar'), 7.80 (d, 1H, C-7' of $\mathrm{Ar}^{\prime}$ ), 6.84 (s, 1H, C-2' of Ar'), 4.10 (s, $2 \mathrm{H}, \mathrm{CH}_{2}$ of methylene), 3.84 (s, $6 \mathrm{H}, \mathrm{OCH}_{3}$ in $\mathrm{Ar}$ ), 3.76 (s, $3 \mathrm{H}, \mathrm{OCH}_{3}$ in $\left.\mathrm{Ar}^{\prime}\right), 10.10$ (s, $1 \mathrm{H}, \mathrm{NH}$ of $\mathrm{Ar}^{\prime}$ ); $\mathrm{MS} \mathrm{m} / \mathrm{z}: 421 \mathrm{M}^{+}$; Anal. Calcd. (\%) for $\mathrm{C}_{21} \mathrm{H}_{19} \mathrm{~N}_{5} \mathrm{O}_{3} \mathrm{~S}$ : C, 59.84; H, 4.54; N, 16.62; S, 7.61. Found: C, 59.72; H, 4.56; N, 16.56; S, 7.64.

3-(3, 4, 5-trimethoxy phenyl)-6-(5-methoxy-3-indolyl methyl)-1,2,4-triazolo[3,4-b]1,3,4-thiadiazole (10c)

Yield: $54 \%$; m.p: $168^{\circ} \mathrm{C}$; IR $(\mathrm{KBr}) \vee\left(\mathrm{cm}^{-1}\right)$ : 3063 (aromatic $\mathrm{CH}$ stretching), $1616(\mathrm{C}=\mathrm{N}$ stretching), 1587, 1549, 1479, 1452 (C=C ring stretch), 2964, 2838 (methyl $\mathrm{CH}$ stretch), 1267 (asymmetric C-O-C stretching), 1029 (symmetric C-O-C stretching), 1280 (N-N=C); ${ }^{1} \mathrm{H}$ NMR $\delta$ (ppm): 7.32 (s, 2H, C-2 \& C-6 of Ar), 7.46 (m, 2H, C-4' \& C-6' of Ar'), 7.78 (d, 1H, C-7' of Ar'), 6.80 (s, 1H, C-2' of Ar'), 4.10 (s, 2H, $\mathrm{CH}_{2}$ of methylene), 3.86 (s, 9H, $\mathrm{OCH}_{3}$ in $\mathrm{Ar}$ ), 3.76 (s, $3 \mathrm{H}, \mathrm{OCH}_{3}$ in $\mathrm{Ar}$ '), 10.10 (s, $1 \mathrm{H}, \mathrm{NH}$ of $\mathrm{Ar}$ '); $\mathrm{MS} \mathrm{m} / z: 451 \mathrm{M}^{+}$; Anal. Calcd. (\%) for $\mathrm{C}_{22} \mathrm{H}_{21} \mathrm{~N}_{5}$ $\mathrm{O}_{4} \mathrm{~S}: \mathrm{C}, 58.52 ; \mathrm{H}, 4.69 ; \mathrm{N}, 15.51 ; \mathrm{S}, 7.10$. Found: C, 58.41; H, 4.71; N, 15.46; S, 7.06.

\section{3-(4-pyridinyl)-6-(5-methoxy-3-indolylmethyl)-1,2,4-triazolo[3,4-b]-1,3,4-thiadiazole(10d)}

Yield: $46 \%$; m.p: $224^{\circ} \mathrm{C}$; IR $(\mathrm{KBr}) \vee\left(\mathrm{cm}^{-1}\right)$ : 3080 (aromatic $\mathrm{CH}$ stretching), $1611(\mathrm{C}=\mathrm{N}$ stretching), 1580, $1560\left(\mathrm{C}=\mathrm{C}\right.$ ring stretch), $1286(\mathrm{~N}-\mathrm{N}=\mathrm{C}) ;{ }^{1} \mathrm{H}$ NMR $\delta(\mathrm{ppm}): 8.0(\mathrm{~d}, 2 \mathrm{H}, \mathrm{C}-$ 3 \& C-5 of Ar), 8.72 (d, 2H, C-2 \& C-6 of Ar), 7.42 (m, 2H, C-4' \& C-6' of Ar'), 7.78 (d, $1 \mathrm{H}, \mathrm{C}-7$ ' of Ar'), 6.80 (s, 1H, C-2 of pyrrole), 4.12 (s, $2 \mathrm{H}, \mathrm{CH}_{2}$ of methylene), 3.76 (s, $3 \mathrm{H}$, $\mathrm{OCH}_{3}$ ), 10.08 (s, $1 \mathrm{H}, \mathrm{NH}$ of Ar'); MS m/z: $362 \mathrm{M}^{+}$; Anal. Calcd. (\%) for $\mathrm{C}_{18} \mathrm{H}_{14} \mathrm{~N}_{6} \mathrm{O} \mathrm{S}: \mathrm{C}$, 59.66; H, 3.89; N, 23.19; S, 8.85. Found: C, 59.54; H, 3.90; N, 23.16; S, 8.81.

3-(3, 4-dimethoxy phenyl)-6-(5-methoxy-2-methyl-3-indolyl methyl)-1,2,4triazolo[3,4-b]-1,3,4-thiadiazole (11a)

Yield: $50 \%$; m.p: $190^{\circ} \mathrm{C}$; IR $(\mathrm{KBr}) \vee\left(\mathrm{cm}^{-1}\right): 3075$ (aromatic $\mathrm{CH}$ stretching), $1610(\mathrm{C}=\mathrm{N}$ stretching), 1587, 1565 ( $\mathrm{C}=\mathrm{C}$ ring stretch), 2980, 2849 (methyl $\mathrm{CH}$ stretch), 1265 (asymmetric CO-C stretching), 1018 (symmetric C-O-C stretching), 1282 (N-N=C); ${ }^{1} \mathrm{H}$ NMR $\delta$ (ppm): 7.18 (d, 1H, C-5 of Ar), 7.56 (s, 1H, C-2 of Ar), 7.72 (d, 1H, C-6 of Ar), 7.44 (d, 1H, C-7' of Ar'), 7.00 (s, 1H, C-4' of Ar'), 7.32 (d, 1H, C-6' of Ar'), 3.80 (s, 3H, $\mathrm{OCH}_{3}$ of $\mathrm{Ar}$ ), 3.76 (s, 6H, $\mathrm{OCH}_{3}$ of $\mathrm{Ar}^{\prime}$ ), 4.10 (s, $2 \mathrm{H}, \mathrm{CH}_{2}$ ), 10.10 (s, $1 \mathrm{H}, \mathrm{NH}$ of $\mathrm{Ar}^{\prime}$ ), 2.84 (s, 3H, $\mathrm{CH}_{3}$ ); MS m/z: $435 \mathrm{M}^{+}$; Anal. Calcd. (\%) for $\mathrm{C}_{22} \mathrm{H}_{21} \mathrm{~N}_{5} \mathrm{O}_{3} \mathrm{~S}: \mathrm{C}, 60.67 ; \mathrm{H}, 4.86 ; \mathrm{N}, 16.08 ; \mathrm{S}, 7.36$. Found: C, 60.79; H, 4.83; N, 16.14; S, 7.31.

3-(3, 5-dimethoxy phenyl)-6-(5-methoxy-2-methyl-3-indolyl methyl)-1,2,4triazolo[3,4-b]-1,3,4-thiadiazole (11b)

Yield: $47 \%$; m.p: $182^{\circ} \mathrm{C}$; IR (KBr) $\vee\left(\mathrm{cm}^{-1}\right)$ : 3082 (aromatic $\mathrm{CH}$ stretching), 1610 (C=N stretching), 1588, 1561 (C=C ring stretch), 2956, 2856 (methyl CH stretch), 1268 (asymmetric C-O-C stretching), 1024 (symmetric C-O-C stretching), $1286(\mathrm{~N}-\mathrm{N}=\mathrm{C}) ;{ }^{1} \mathrm{H}$ NMR $\delta(\mathrm{ppm}): 7.20$ (d, 2H, C-2 \& C-6 of Ar), 6.74 (d, 1H, C-4 of Ar), 7.48 (d, 1H, C-7' of Ar'), 7.00 (s, 1H, C-4' of Ar'), 7.34 (d, 1H, C-6' of $\mathrm{Ar}$ '), 4.10 (s, $2 \mathrm{H}, \mathrm{CH}_{2}$ of methylene), 3.82 (s, $6 \mathrm{H}, \mathrm{OCH}_{3}$ in $\mathrm{Ar}$ ), 3.76 (s, $3 \mathrm{H}, \mathrm{OCH}_{3}$ in $\left.\mathrm{Ar}^{\prime}\right), 10.10$ (s, 1H, NH of Ar'), 2.84 (s, 3H, $\mathrm{CH}_{3}$ ); MS m/z: $435 \mathrm{M}^{+}$; Anal. Calcd. (\%) for $\mathrm{C}_{22} \mathrm{H}_{21} \mathrm{~N}_{5} \mathrm{O}_{3} \mathrm{~S}: \mathrm{C}, 60.67$; H, 4.86; N, 16.08; S, 7.36. Found: C, 60.72; H, 4.84; N, 16.12; S, 7.33. 
3-(3, 4, 5-trimethoxy phenyl)-6-(5-methoxy-2-methyl-3-indolyl methyl)-1,2,4triazolo [3,4-b] -1,3,4-thiadiazole (11c)

Yield: $50 \%$; m.p: $186^{\circ} \mathrm{C}$; IR (KBr) $\vee\left(\mathrm{cm}^{-1}\right)$ : 3068 (aromatic $\mathrm{CH}$ stretching), $1616(\mathrm{C}=\mathrm{N}$ stretching), 1587, 1554 (C=C ring stretch), 2943, 2860 (methyl $\mathrm{CH}$ stretch), 1258 (asymmetric CO-C stretching), 1023 (symmetric C-O-C stretching), 1288 (N-N=C); ${ }^{1} \mathrm{H}$ NMR $\delta$ (ppm): 7.20 (s, 2H C-2 \& C-6 of Ar), 7.58 (d, 1H, C-7' of Ar'), 6.96 (s, 1H, C-4' of Ar'), 7.36 (d, 1H, C-6' of Ar'), 4.24 (s, $2 \mathrm{H}, \mathrm{CH}_{2}$ of methylene), $3.8\left(\mathrm{~s}, 9 \mathrm{H}, \mathrm{OCH}_{3}\right.$ in $\left.\mathrm{Ar}\right), 3.76\left(\mathrm{~s}, 3 \mathrm{H}, \mathrm{OCH}_{3}\right.$ in $\left.\mathrm{Ar}^{\prime}\right), 10.08(\mathrm{~s}, 1 \mathrm{H}$, $\mathrm{NH}$ of Ar'), 2.84 (s, 3H, $\mathrm{CH}_{3}$ ); MS m/z: $465 \mathrm{M}^{+}$; Anal. Calcd. (\%) for $\mathrm{C}_{23} \mathrm{H}_{23} \mathrm{~N}_{5} \mathrm{O}_{4} \mathrm{~S}: \mathrm{C}, 59.34 ; \mathrm{H}$, 4.98; N, 15.04; S, 6.89. Found: C, 59.46; H, 4.97; N, 15.09; S, 6.85.

3-(4-pyridinyl)-6-(5-methoxy-2-methyl-3-indolyl methyl)-1,2,4-triazolo[3,4-b]1,3,4-thiadiazole (11d)

Yield: $53 \%$; m.p: $240^{\circ} \mathrm{C}$; IR $(\mathrm{KBr}) \vee\left(\mathrm{cm}^{-1}\right)$ : 3080 (aromatic $\mathrm{CH}$ stretching), $1611(\mathrm{C}=\mathrm{N}$ stretching), 1580, $1560\left(\mathrm{C}=\mathrm{C}\right.$ ring stretch), $1286(\mathrm{~N}-\mathrm{N}=\mathrm{C}) ;{ }^{1} \mathrm{H}$ NMR $\delta(\mathrm{ppm}): 8.0(\mathrm{~d}, 2 \mathrm{H}, \mathrm{C}-$ 3 \& C-5 of Ar), 8.72 (d, 2H, C-2 \& C-6 of Ar), 7.00 (s, 1H, C-4' of Ar'), 7.28 (d, 1H, C-6' of Ar'), 7.56 (d, 1H, C-7' of Ar'), 4.10 (s, 2H, $\mathrm{CH}_{2}$ of methylene), 3.76 (s, 3H, $\mathrm{OCH}_{3}$ in Ar'), 10.06 (s, $1 \mathrm{H}, \mathrm{NH}$ of Ar'), 2.84 (s, 3H, $\mathrm{CH}_{3}$ ); MS m/z: $376 \mathrm{M}^{+}$; Anal. Calcd. (\%) for $\mathrm{C}_{19}$ $\mathrm{H}_{16} \mathrm{~N}_{6}$ O S: C, 60.62; H, 4.28; N, 22.33; S, 8.52. Found: C, 60.56; H, 4.30; N, 22.27; S, 8.49.

3-(3, 4-dimethoxy phenyl)-6-(3-indolyl methyl)-1,2,4-triazolo[3,4-b]-1,3,4thiadiazole (12a)

Yield: $58 \%$; m.p: $184^{\circ} \mathrm{C}$; IR $(\mathrm{KBr}) \vee\left(\mathrm{cm}^{-1}\right)$ : 3092 (aromatic $\mathrm{CH}$ stretching), $1623(\mathrm{C}=\mathrm{N}$ stretching), 1583, 1545 ( $\mathrm{C}=\mathrm{C}$ ring stretch), 2980, 2855 (methyl $\mathrm{CH}$ stretch), 1267 (asymmetric C-O-C stretching), 1018 (symmetric C-O-C stretching), $1282(\mathrm{~N}-\mathrm{N}=\mathrm{C}) ;{ }^{1} \mathrm{H}$ NMR $\delta$ (ppm): 7.1 (d, 1H C-5 of Ar), 7.54 (s, 1H, C-2 of Ar), 7.70 (d, 1H, C-6 of Ar), 7.20 (s, 4H, C-4, C-5, C-6 \& C-7 of Ar'), 6.8 (s, $1 \mathrm{H}$ of C-2 of Ar'), 3.80 (s, $6 \mathrm{H}, \mathrm{OCH}_{3}$ in Ar), $4.20\left(\mathrm{~s}, 2 \mathrm{H}, \mathrm{CH}_{2}\right), 10.08$ (s, $1 \mathrm{H}, \mathrm{NH}$ of $\mathrm{Ar}$ '); MS m/z: $391 \mathrm{M}^{+}$; Anal. Calcd. (\%) for $\mathrm{C}_{20} \mathrm{H}_{17}$ $\mathrm{N}_{5} \mathrm{O}_{2} \mathrm{~S}$ : C, 61.37; H, 4.38; N, 17.89; S, 8.19. Found: C, 61.26; H, 4.41; N, 17.82; S, 8.15.

3-(3, 5-dimethoxy phenyl)-6-(3-indolyl methyl)-1,2,4-triazolo[3,4-b]-1,3,4thiadiazole (12b)

Yield: $58 \%$; m.p: $174^{\circ} \mathrm{C}$; IR (KBr) $\vee\left(\mathrm{cm}^{-1}\right)$ : 3071 (aromatic $\mathrm{CH}$ stretching), 1608 (C=N stretching), 1574, 1540 (C=C ring stretch), 2956, 2834 (methyl CH stretch), 1261 (asymmetric C-O-C stretching), 1019 (symmetric C-O-C stretching), $1280(\mathrm{~N}-\mathrm{N}=\mathrm{C}) ;{ }^{1} \mathrm{H}$ NMR $\delta$ (ppm): 7.28 (d, 2H, C-2 \& C-6 of $\mathrm{Ar}$ ), 6.64 (d, 1H, C-4 of Ar), 7.20 (s, 4H, C-4, C-5, C-6 \& C-7 of Ar'), 6.8 (s, 1H of C-2 of Ar'), 4.10 (s, $2 \mathrm{H}, \mathrm{CH}_{2}$ ), 10.08 (s, $1 \mathrm{H}, \mathrm{NH}$ of Ar'), 3.84 (s, 6H, $\mathrm{OCH}_{3}$ in $\mathrm{Ar}$ ); $\mathrm{MS} \mathrm{m} / z: 391 \mathrm{M}^{+}$; Anal. Calcd. (\%) for $\mathrm{C}_{20} \mathrm{H}_{17} \mathrm{~N}_{5} \mathrm{O}_{2} \mathrm{~S}: \mathrm{C}, 61.37 ; \mathrm{H}, 4.38 ; \mathrm{N}, 17.89 ; \mathrm{S}$, 8.19. Found: C, 61.33; H, 4.38; N, 17.86; S, 8.16.

3-(3, 4, 5-trimethoxy phenyl)-6-(3-indolyl methyl)-1,2,4-triazolo[3,4-b]-1,3,4thiadiazole (12c)

Yield: $50 \%$; m.p: $180^{\circ} \mathrm{C}$; IR (KBr) $\vee\left(\mathrm{cm}^{-1}\right)$ : 3060 (aromatic CH stretching), 1616 (C=N stretching), 1571, 1549 (C=C ring stretch), 2952, 2839 (methyl CH stretch), 1259 (asymmetric C-O-C stretching), 1022 (symmetric C-O-C stretching), $1271(\mathrm{~N}-\mathrm{N}=\mathrm{C}) ;{ }^{1} \mathrm{H}$ NMR $\delta$ (ppm): 7.36 (s, 2H, C-2 \& C-6 of Ar), 7.20 (s, 4H, C-4, C-5, C-6 \& C-7 of Ar'), 6.8 (s, 1H of C-2 of Ar'), 4.10 (s, 2H, $\mathrm{CH}_{2}$ ), 10.08 (s, $1 \mathrm{H}, \mathrm{NH}$ of $\mathrm{Ar}$ '), 3.86 (s, 9H, $\mathrm{OCH}_{3}$ of $\mathrm{Ar}$ ); $\mathrm{MS} m / z: 421 \mathrm{M}^{+}$; Anal. Calcd. (\%) for $\mathrm{C}_{21} \mathrm{H}_{19} \mathrm{~N}_{5} \mathrm{O}_{3} \mathrm{~S}: \mathrm{C}$, 59.84; H, 4.54; N, 16.62; S, 7.61. Found: C, 59.73; H, 4.57; N, 16.65; S, 7.58. 
3-(4-pyridinyl)-6-(3-indolyl methyl)-1,2,4-triazolo[3,4-b]-1,3,4-thiadiazole (12d)

Yield: $55 \%$; m.p: $232^{\circ} \mathrm{C}$; $\mathrm{IR}(\mathrm{KBr}) \vee\left(\mathrm{cm}^{-1}\right)$ : 3080 (aromatic $\mathrm{CH}$ stretching), $1611(\mathrm{C}=\mathrm{N}$ stretching), 1580, $1560\left(\mathrm{C}=\mathrm{C}\right.$ ring stretch), $1286(\mathrm{~N}-\mathrm{N}=\mathrm{C}) ;{ }^{1} \mathrm{H}$ NMR $\delta(\mathrm{ppm}): 8.0(\mathrm{~d}, 1 \mathrm{H}, \mathrm{C}-3$ \& C-5 of Ar), 8.7 (d, 1H, C-2 \& C-6 of Ar), 7.20 (s, 4H, C-4, C-5, C-6 \& C-7 of Ar'), 6.8 (s, 1H of $\mathrm{C}-2$ of $\mathrm{Ar}$ '), 4.10 (s, 2H, $\mathrm{CH}_{2}$ ), 10.18 (s, $1 \mathrm{H}, \mathrm{NH}$ of Ar'); MS m/z: $332 \mathrm{M}^{+}$; Anal. Calcd. (\%) for $\mathrm{C}_{17} \mathrm{H}_{12} \mathrm{~N}_{6} \mathrm{~S}: \mathrm{C}, 61.43 ; \mathrm{H}, 3.64 ; \mathrm{N}, 25.28 ; \mathrm{S}, 9.65$. Found: C, 61.34; H, 3.66; N, 25.22; S, 9.61.

3-(3,4-dimethoxy phenyl)-6-(4-hydroxy-3-methoxy cinnamyl)-1,2,4-triazolo [3,4-b]1,3,4-thiadiazole (13a)

Yield: 62\%; m.p: $194{ }^{\circ} \mathrm{C}$; IR (KBr) $\vee\left(\mathrm{cm}^{-1}\right)$ : 3435 (OH stretching), 3036 (aromatic $\mathrm{CH}$ stretching), 1614 ( $\mathrm{C}=\mathrm{N}$ stretching), 1590, 1549, 1477, 1453 (C=C ring stretch), 2977, 2840 (methyl CH stretch), 1265 (asymmetric C-O-C stretching), 1023 (symmetric C-O-C stretching), $1280(\mathrm{~N}-\mathrm{N}=\mathrm{C}), 1636$ (vinyl group); 1H NMR $\delta(\mathrm{ppm}): 7.16$ (d, 1H C-5 of Ar), 7.56 (s, 1H, C-2 of Ar), 7.68 (d, 1H, C-6 of Ar), 7.76 (s, 1H, C-2 of Ar'), 7.42 (d, 1H, C-5 of $\left.\mathrm{Ar}^{\prime}\right), 7.8\left(\mathrm{~d}, 1 \mathrm{H}, \mathrm{C}-6\right.$ of Ar'), 3.74 (s, 3H, $\mathrm{OCH}_{3}$ of Ar'), 3.84 (s, 6H, $\mathrm{OCH}_{3}$ of $\mathrm{Ar}$ ), 6.96 (d, $1 \mathrm{H}, \mathrm{CH}), 6.8(\mathrm{~d}, 1 \mathrm{H}, \mathrm{CH}), 6.14(\mathrm{~s}, 1 \mathrm{H}, \mathrm{OH})$; MS $m / z: 410 \mathrm{M}^{+}$; Anal. Calcd. (\%) for $\mathrm{C}_{20} \mathrm{H}_{18}$ $\mathrm{N}_{4} \mathrm{O}_{4} \mathrm{~S}:$ C, 58.52; H, 4.42; N, 13.65; S, 7.81. Found: C, 58.43; H, 4.41; N, 13.68; S, 7.78.

3-(3,5-dimethoxy phenyl)-6-(4-hydroxy-3-methoxy cinnamyl)-1,2,4-triazolo[3,4-b]1,3,4-thiadiazole (13b)

Yield: 62\%; m.p: $188^{\circ} \mathrm{C}$; $\mathrm{IR}(\mathrm{KBr}) \vee\left(\mathrm{cm}^{-1}\right): 3443$ (OH stretching), 3040 (aromatic $\mathrm{CH}$ stretching), 1614 (C=N stretching), 1588, 1541, 1477, 1452 (C=C ring stretch), 2956, 2909 (methyl CH stretch), 1260 (asymmetric C-O-C stretching), 1019 (symmetric C-O-C stretching), 1290 (N-N=C), 1636 (vinyl group); ${ }^{1} \mathrm{H}$ NMR $\delta$ (ppm): 7.24 (d, 2H, C-2 \& C-6 of Ar), 6.64 (d, 1H, C-4 of Ar), 7.72 (s, 1H, C-2 of Ar'), 7.46 (d, 1H, C-4 of Ar'), 7.8 (d, 1H, C-6 of Ar'), 6.96 (d, 1H, CH), $6.8(\mathrm{~d}, 1 \mathrm{H}, \mathrm{CH}), 3.74$ (s, $3 \mathrm{H}, \mathrm{OCH}_{3}$ of Ar'), 3.84 (s, 6H, $\mathrm{OCH}_{3}$ of Ar), 6.14 (s, $1 \mathrm{H}, \mathrm{OH}$ ); $\mathrm{MS} \mathrm{m} / z: 410 \mathrm{M}^{+}$; Anal. Calcd. (\%) for $\mathrm{C}_{20} \mathrm{H}_{18} \mathrm{~N}_{4} \mathrm{O}_{4} \mathrm{~S}: \mathrm{C}, 58.52 ; \mathrm{H}, 4.42 ; \mathrm{N}, 13.65 ; \mathrm{S}, 7.81$. Found: $\mathrm{C}, 58.44 ; \mathrm{H}, 4.43 ; \mathrm{N}, 13.61 ; \mathrm{S}, 7.84$.

3-(3,4,5-trimethoxy phenyl)-6-(4-hydroxy-3-methoxy cinnamyl)-1,2,4-triazolo[3,4b]-1,3,4-thiadiazole (13c)

Yield: 60\%; m.p: 146-148 ${ }^{\circ} \mathrm{C}$; IR (KBr) v $\left(\mathrm{cm}^{-1}\right)$ : 3437 (OH stretching), 3047 (aromatic $\mathrm{CH}$ stretching), 1616 (C=N stretching), 1587, 1549, 1479, 1452 (C=C ring stretch), 2927 (methyl CH stretch), 1263 (asymmetric C-O-C stretching), 1017 (symmetric C-O-C stretching), 1280 (N-N=C), 1640 (vinyl group); ${ }^{1} \mathrm{H}$ NMR $\delta$ (ppm): 7.30 (s, 2H, C-2 \& C-6 of Ar), 7.74 (s, 1H, C-2 of Ar'), 7.46 (d, 1H, C-4 of Ar), $7.8\left(\mathrm{~d}, 1 \mathrm{H}, \mathrm{C}-6\right.$ of Ar), $7.0(\mathrm{~d}, 1 \mathrm{H}, \mathrm{CH}), 6.86(\mathrm{~d}, 1 \mathrm{H}, \mathrm{CH}), 3.74\left(\mathrm{~s}, 3 \mathrm{H}, \mathrm{OCH}_{3}\right.$ of $\mathrm{Ar}^{\prime}$ ), 3.88 (s, 9H, $\mathrm{OCH}_{3}$ of $\mathrm{Ar}$ ), $6.12(\mathrm{~s}, 1 \mathrm{H}, \mathrm{OH})$; $\mathrm{MS} m / z: 440 \mathrm{M}^{+}$; Anal. Calcd. (\%) for $\mathrm{C}_{21} \mathrm{H}_{20} \mathrm{~N}_{4}$ $\mathrm{O}_{5}$ S: C, 57.26; H, 4.58; N, 12.72; S, 7.28. Found: C, 57.35; H, 4.54; N, 12.67; S, 7.23.

3-(4-pyridinyl)-6-(4-hydroxy-3-methoxy cinnamyl)-1,2,4-triazolo[3,4-b]-1,3,4thiadiazole (13d)

Yield: 64\%; m.p: $210{ }^{\circ} \mathrm{C}$; IR (KBr) $\vee\left(\mathrm{cm}^{-1}\right): 3445$ (OH stretching), 3069 (aromatic $\mathrm{CH}$ stretching), $1620(\mathrm{C}=\mathrm{N}$ stretching), 1578, $1554(\mathrm{C}=\mathrm{C}$ ring stretch), 2960, 2844 (methyl $\mathrm{CH}$ stretch), $1276(\mathrm{~N}-$ $\mathrm{N}=\mathrm{C}), 1636$ (vinyl group); ${ }^{1} \mathrm{H}$ NMR $\delta$ (ppm): 8.0 (d, 1H, C-3 \& C-5 of Ar), 8.7 (d, 1H, C-2 \& C-6 of $\mathrm{Ar}), 7.74$ (s, 1H, C-2 of Ar'), 7.46 (d, 1H, C-4 of Ar), 7.8 (d, 1H, C-6 of Ar), 7.00 (d, 1H, CH) 6.88, (d, $1 \mathrm{H}, \mathrm{CH}), 6.14$ (s, $1 \mathrm{H}, \mathrm{OH}), 3.74$ (3s, 3H, $\mathrm{OCH}_{3}$ in Ar'); MS m/z: $351 \mathrm{M}^{+}$; Anal. Calcd. (\%) for $\mathrm{C}_{17} \mathrm{H}_{13}$ $\mathrm{N}_{5} \mathrm{O}_{2} \mathrm{~S}: \mathrm{C}, 58.11 ; \mathrm{H}, 3.73 ; \mathrm{N}, 19.93 ; \mathrm{S}, 9.13$. Found: C, 57.97; H, 3.71; N, 19.97; S, 9.10. 
3-(3,4-dimethoxy phenyl)-6-(2-amino-3,5-dibromo phenyl)-1,2,4-triazolo[3,4-b]1,3,4-thiadiazole (14a)

Yield:46\%; m.p: 242-246 ${ }^{\circ} \mathrm{C}$; IR (KBr) v $\left(\mathrm{cm}^{-1}\right)$ : 3290 (NH stretching), 3190, 3060 (aromatic CH stretching), 1614 (C=N stretching), 1584, 1553, 1482, 1456 (C=C ring stretch), 2958, 2932 (methyl CH stretch), 1264 (asymmetric C-O-C stretching), 1020 (symmetric C-O-C stretching), $1280(\mathrm{~N}-\mathrm{N}=\mathrm{C}) ;{ }^{1} \mathrm{H}$ NMR $\delta$ (ppm): 7.14 (d, 1H C-5 of Ar), 7.56 (s, 1H, C-2 of Ar), 7.68 (d, 1H, C-6 of Ar), 7.88 (s, 1H, C-4 of Ar'), 7.96 (s, 1H, C-6 of Ar'), 6.28 (s, 2H, NH $\left.\mathrm{N}_{2}\right), 3.80$ (s, 6H, $\mathrm{OCH}_{3}$ ); MS $m / z: 511 \mathrm{M}^{+}, 513 \mathrm{M}^{+2}, 515 \mathrm{M}^{+4}$; Anal. Calcd. (\%) for $\mathrm{C}_{17} \mathrm{H}_{13} \mathrm{~N}_{5} \mathrm{O}_{2} \mathrm{~S} \mathrm{Br}_{2}$ : C, 39.94; H, 2.56; N, 13.70; S, 6.27. Found: C, 39.84; H, 2.59; N, 13.65; S, 6.29.

3-(3,5-dimethoxy phenyl)-6-(2-amino-3, 5-dibromo phenyl)-1,2,4-triazolo [3,4-b] 1,3,4-thiadiazole (14b)

Yield: 48\%; m.p: $228{ }^{\circ} \mathrm{C}$; IR (KBr) $\vee\left(\mathrm{cm}^{-1}\right)$ : 3298 (NH stretching), 3140, 3074 (aromatic $\mathrm{CH}$ stretching), $1610(\mathrm{C}=\mathrm{N}$ stretching), 1590, 1570, 1490, 1465 (C=C ring stretch), 2960 (methyl CH stretch), 1262 (asymmetric C-O-C stretching), 1019 (symmetric C-O-C stretching), $1283(\mathrm{~N}-\mathrm{N}=\mathrm{C}) ;{ }^{1} \mathrm{H}$ NMR $\delta$ (ppm): 7.24 (d, 2H, C-2 \& C-6 of Ar), $6.68(\mathrm{~d}, 1 \mathrm{H}$, C-4 of Ar), 7.88 (s, 1H, C-4 of Ar'), 7.96 (s, 1H, C-6 of Ar'), 6.28 (s, 2H, NH $\left.\mathrm{N}_{2}\right), 3.84$ (s, 6H, $\mathrm{OCH}_{3}$ ); MS $m / z: 511 \mathrm{M}^{+}, 513 \mathrm{M}^{+2}, 515 \mathrm{M}^{+4}$; Anal. Calcd. (\%) for $\mathrm{C}_{17} \mathrm{H}_{13} \mathrm{~N}_{5} \mathrm{O}_{2} \mathrm{~S} \mathrm{Br}_{2}: \mathrm{C}$, 39.94; H, 2.56; N, 13.70; S, 6.27. Found: C, 39.86; H, 2.56; N, 13.68; S, 6.24.

3-(3,4,5-trimethoxy phenyl)-6-(2-amino-3, 5-dibromo phenyl)-1,2,4-triazolo[3,4-b]1,3,4-thiadiazole (14c)

Yield: 48\%; m.p: $202{ }^{\circ} \mathrm{C}$; IR (KBr) $\vee\left(\mathrm{cm}^{-1}\right): 3273$ (NH stretching), 3090, 3047 (aromatic $\mathrm{CH}$ stretching), 1612 (C=N stretching), 1585, 1575, 1484, 1436 (C=C ring stretch), 2964 (methyl CH stretch), 1290 (N-N=C); 1H NMR $\delta$ (ppm): 7.32 (s, 2H, C-2 \& C-6 of Ar), 7.86 (s, 1H, C-4 of Ar'), 7.94 (s, 1H, C-6 of Ar'), 6.28 (s, 2H, NH $\mathrm{NH}_{2}, 3.88$ (s, 9H, $\left.\mathrm{OCH}_{3}\right)$; MS m/z: $541 \mathrm{M}^{+}, 543 \mathrm{M}^{+2}, 545 \mathrm{M}^{+4}$; Anal. Calcd. (\%) for $\mathrm{C}_{18} \mathrm{H}_{15} \mathrm{~N}_{5} \mathrm{O}_{3} \mathrm{~S} \mathrm{Br}_{2}: \mathrm{C}, 39.95 ; \mathrm{H}, 2.79 ; \mathrm{N}$, 12.94; S, 5.92. Found: C, 40.07; H, 2.81; N, 12.86; S, 5.87.

3-(4-pyridinyl)-6-(2-amino-3,5-dibromophenyl)-1,2,4-triazolo[3,4-b]-1,3,4thiadiazole $(14 d)$

Yield: 43\%; m.p: $214{ }^{\circ} \mathrm{C} ; \mathrm{IR}(\mathrm{KBr}) \vee\left(\mathrm{cm}^{-1}\right): 3280$ (NH stretching), 3074 (aromatic $\mathrm{CH}$ stretching), $1618\left(\mathrm{C}=\mathrm{N}\right.$ stretching), 1585, $1554\left(\mathrm{C}=\mathrm{C}\right.$ ring stretch), $1280(\mathrm{~N}-\mathrm{N}=\mathrm{C}) ;{ }^{1} \mathrm{H}$ NMR $\delta(\mathrm{ppm}): 8.04$ (d, $1 \mathrm{H}, \mathrm{C}-3$ \& C-5 of Ar), 8.7 (d, 1H, C-2 \& C-6 of Ar), 7.86 (s, 1H, C-4 of Ar'), 7.92 (s, 1H, C-6 of $\left.\mathrm{Ar}^{\prime}\right), 6.28\left(\mathrm{~s}, 2 \mathrm{H}, \mathrm{NH}_{2}\right.$ ); $\mathrm{MS} m / z: 452 \mathrm{M}^{+}, 454 \mathrm{M}^{+2}, 456 \mathrm{M}^{+4}$; Anal. Calcd. (\%) for $\mathrm{C}_{14} \mathrm{H}_{8} \mathrm{~N}_{6} \mathrm{~S} \mathrm{Br}_{2}$ : C, 37.19; H, 1.78; N, 18.59; S, 7.09. Found: C, 37.12; H, 1.79; N, 18.54; S, 7.69.

3-(3,4-dimethoxy phenyl)-6-(2-nitro-4-methyl phenyl)-1,2,4-triazolo [3,4-b]-1,3,4thiadiazole (15a)

Yield: 65\%; m.p: 194-196 ${ }^{\circ} \mathrm{C}$; IR (KBr) $v\left(\mathrm{~cm}^{-1}\right)$ : 3076 (aromatic $\mathrm{CH}$ stretching), $1613(\mathrm{C}=\mathrm{N}$ stretching), 1586, 1485, 1451 (C=C ring stretch), 2966, 2933 (methyl CH stretch), 1266 (asymmetric C-O-C stretching), 1021 (symmetric C-O-C stretching), 1284 (N-N=C), 1525 (asymmetric $\mathrm{Ar}^{-\mathrm{NO}_{2}}$ stretch), 1346 (symmetric Ar-NO ${ }_{2}$ stretch; ${ }^{1} \mathrm{H}$ NMR $\delta$ (ppm): 7.12 (d, 1H, C-5 of Ar), 7.54 (s, 1H, C-2 of Ar), 7.70 (d, 1H, C-6 of Ar), 8.22 (s, 1H, C-3 of Ar'), 7.84 ( d, 1H, C-5 of Ar'), 7.95 ( d, 1H, C-6 of $\left.\mathrm{Ar}^{\prime}\right), 3.80\left(\mathrm{~s}, 6 \mathrm{H}, \mathrm{OCH}_{3}\right), 2.82$ (s, $3 \mathrm{H}, \mathrm{CH}_{3}$ of Ar'); MS m/z: $397 \mathrm{M}^{+}$; Anal. Calcd. (\%) For $\mathrm{C}_{18} \mathrm{H}_{15} \mathrm{~N}_{5}$ $\mathrm{O}_{4}$ S: C, 54.40; H, 3.80; N, 17.62; S, 8.07. Found: C, 54.32; H, 3.82; N, 17.58; S, 8.10. 
3-(3,5-dimethoxy phenyl)-6-(2-nitro-4-methyl phenyl)-1,2,4-triazolo [3,4-b]-1,3,4thiadiazole (15b)

Yield: 67\%; m.p: $187{ }^{\circ} \mathrm{C}$; IR $(\mathrm{KBr}) \vee\left(\mathrm{cm}^{-1}\right)$ : 3075 (aromatic $\mathrm{CH}$ stretching), 1609 (C=N stretching), 1586, 1554, 1484, 1453 (C=C ring stretch), 2963, 2930 (methyl $\mathrm{CH}$ stretch), 1265 (asymmetric C-O-C stretching), 1027 (symmetric C-O-C stretching), 1286 (N-N=C), 1524 (asymmetric $\mathrm{Ar}-\mathrm{NO}_{2}$ stretch), 1345 (symmetric $\mathrm{Ar}-\mathrm{NO}_{2}$ stretch); ${ }^{1} \mathrm{H} \mathrm{NMR} \delta$ (ppm): 7.28 (d, 2H, C2 \& C-6 of Ar), 6.68 (d, 1H, C-4 of Ar), 8.22 (s, 1H, C-3 of Ar'), 7.84 ( d, 1H, C-5 of Ar'), 7.95 ( d,

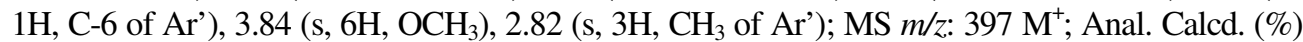
for $\mathrm{C}_{18} \mathrm{H}_{15} \mathrm{~N}_{5} \mathrm{O}_{4} \mathrm{~S}: \mathrm{C}, 54.40 ; \mathrm{H}, 3.80 ; \mathrm{N}, 17.62 ; \mathrm{S}$, 8.07. Found: C, 54.47; H, 3.78; N, 17.57; S, 8.04.

3-(3,4,5-trimethoxy phenyl)-6-(2-nitro-4-methyl phenyl)-1,2,4-triazolo [3,4-b]1,3,4-thiadiazole (15c)

Yield: 63\%; m.p: $174-176{ }^{\circ} \mathrm{C}$; $\mathrm{IR}(\mathrm{KBr}) \vee\left(\mathrm{cm}^{-1}\right)$ : 3077 (aromatic $\mathrm{CH}$ stretching), $1618(\mathrm{C}=\mathrm{N}$ stretching), 1589, 1554, 1483, 1455 (C=C ring stretch), 1259 (asymmetric C-O-C stretching), 1024

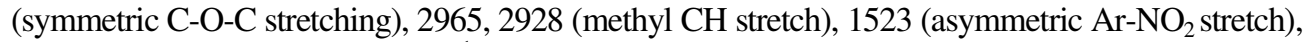
1343 (symmetric Ar-NO ${ }_{2}$ stretch); ${ }^{1} \mathrm{H}$ NMR $\delta$ (ppm): 7.34 (s, 2H, C-2 \& C-6 of Ar), 8.20 (s, 1H, C3 of Ar'), 7.84 ( d, 1H, C-5 of Ar'), 7.96 ( d, 1H, C-6 of Ar'), 3.86 (s, 9H, OCH $\left.\mathrm{OH}_{3}\right), 2.80\left(\mathrm{~s}, 3 \mathrm{H}, \mathrm{CH}_{3}\right.$ in $\mathrm{Ar}^{\prime}$ ); $\mathrm{MS} \mathrm{m} / z$ : $427 \mathrm{M}^{+}$; Anal. Calcd. (\%) for $\mathrm{C}_{19} \mathrm{H}_{17} \mathrm{~N}_{5} \mathrm{O}_{5} \mathrm{~S}: \mathrm{C}, 53.39 ; \mathrm{H}, 4.01 ; \mathrm{N}, 16.38 ; \mathrm{S}, 7.50$. Found: C, 53.32; H, 3.97; N, 16.46; S, 7.47.

\section{3-(4-pyridinyl)-6-(2-nitro-4-methyl phenyl)-1,2,4-triazolo [3,4-b]-1,3,4-thiadiazole (15d)}

Yield: 65\%; m.p: $206{ }^{\circ} \mathrm{C}$; IR (KBr) $v\left(\mathrm{~cm}^{-1}\right)$ : 3085 (aromatic $\mathrm{CH}$ stretching), $1611(\mathrm{C}=\mathrm{N}$ stretching), 1573, 1562 (C=C ring stretch), 2960, 2842 (methyl CH stretch), 1530 (asymmetric Ar$\mathrm{NO}_{2}$ stretch), 1349 (symmetric $\mathrm{Ar}-\mathrm{NO}_{2}$ stretch); ${ }^{1} \mathrm{H} \mathrm{NMR} \delta$ (ppm): 8.12 (d, 1H, C-3 \& C-5 of Ar), 8.86 (d, 1H, C-2 \& C-6 of Ar), 8.22 (s, 1H, C-3 of Ar'), 7.84 ( d, 1H, C-5 of Ar'), 7.94 ( d, 1H, C-6 of Ar'), 7.86 (d, 2H of Ar'), 2.80 (s, 3H, $\mathrm{CH}_{3}$ of Ar'); MS m/z: $338 \mathrm{M}^{+}$; Anal. Calcd. (\%) for $\mathrm{C}_{15} \mathrm{H}_{10}$ $\mathrm{N}_{6} \mathrm{O}_{2} \mathrm{~S}: \mathrm{C}, 53.25 ; \mathrm{H}, 2.98 ; \mathrm{N}, 24.84 ; \mathrm{S}, 9.48$. Found: C, 53.18; H, 2.98; N, 24.76; S, 9.45.

3-(3,4-dimethoxy phenyl)-6-[1-(2,4-dichloro phenoxy) ethyl]-1,2,4-triazolo[3,4-b]1,3,4-thiadiazole (16a)

Yield: 56(\%); m.p: $78{ }^{\circ} \mathrm{C}$; IR $(\mathrm{KBr}) \vee\left(\mathrm{cm}^{-1}\right)$ : 3078 (aromatic $\mathrm{CH}$ stretching), $1612(\mathrm{C}=\mathrm{N}$ stretching), 1585, 1550 (C=C ring stretch), 2954, 2946 (methyl CH stretch), 1260 (asymmetric CO-C stretching), 1022(symmetric C-O-C stretching), 1281 (N-N=C); ${ }^{1} \mathrm{H}$ NMR $\delta$ (ppm): 7.16 (d, 1H, C-5 of Ar), 7.54 (s, 1H, C-2 of Ar), 7.68 (d, 1H, C-6 of Ar), 7.86 (s, 1H, C-3' of Ar), 7.76 (s, $1 \mathrm{H}, \mathrm{C}-5$ ' of $\mathrm{Ar}$ ), 6.92 (d, 1H, C-6' of Ar'), 5.80 (q, 1H, CH in -CH-CH $)_{3}, 1.84$ (d, $3 \mathrm{H}$ of $\mathrm{CH}_{3}$ in CH-CH $\left.\mathrm{CH}_{3}\right), 3.80\left(\mathrm{~s}, 6 \mathrm{H}, \mathrm{OCH}_{3}\right)$; MS $m / z: 451 \mathrm{M}^{+}, 453 \mathrm{M}^{+2}, 455 \mathrm{M}^{+4}$; Anal. Calcd. (\%) for $\mathrm{C}_{19} \mathrm{H}_{16} \mathrm{~N}_{4}$ $\mathrm{O}_{3} \mathrm{~S} \mathrm{Cl}_{2}:$ C, 50.56; H, 3.57; N, 12.41; S, 7.10. Found: C, 50.67; H, 3.59; N, 12.38; S, 7.07.

3-(3,5-dimethoxy phenyl)-6-[1-(2,4-dichloro phenoxy) ethyl]-1,2,4-triazolo [3,4-b]1,3,4-thiadiazole (16b)

Yield: 56\%; m.p: $70{ }^{\circ} \mathrm{C}$; IR ( $\left.\mathrm{KBr}\right) \vee\left(\mathrm{cm}^{-1}\right): 3085$ (aromatic $\mathrm{CH}$ stretching), 1611(C=N stretching), 1581, 1549, 1475, 1439 (C=C ring stretch), 2981, 2934 (methyl CH stretch), 1264 (asymmetric C-O-C stretching), 1028 (symmetric C-O-C stretching), $1280(\mathrm{~N}-\mathrm{N}=\mathrm{C}) ;{ }^{1} \mathrm{H}$ NMR $\delta(\mathrm{ppm}): 7.2$ (d, 2H, of Ar), 6.7 (d, 1H, of Ar), 7.86 (s, 1H, C-3' of Ar), 7.76 (s, 1H, C-5' of Ar), 6.92 (d, 1H, C-6' of Ar'), 5.80 (q, $1 \mathrm{H}, \mathrm{CH}$ in $\left.-\mathrm{CH}-\mathrm{CH}_{3}\right), 1.84\left(\mathrm{~d}, 3 \mathrm{H}\right.$ of $\mathrm{CH}_{3}$ in $\left.-\mathrm{CH}-\mathrm{CH}_{3}\right), 3.84\left(\mathrm{~s}, 6 \mathrm{H}, \mathrm{OCH}_{3}\right) ; \mathrm{MS} \mathrm{m} / z: 451 \mathrm{M}^{+}, 453$ $\mathrm{M}^{+2}$, $455 \mathrm{M}^{+4}$; Anal. Calcd. (\%) for $\mathrm{C}_{19} \mathrm{H}_{16} \mathrm{~N}_{4} \mathrm{O}_{3} \mathrm{~S} \mathrm{Cl}_{2}: \mathrm{C}, 50.56 ; \mathrm{H}, 3.57 ; \mathrm{N}, 12.41 ; \mathrm{S}, 7.10$. Found: $\mathrm{C}$, 50.68; H, 3.56; N, 12.38; S, 7.07. 
3-(3,4,5-trimethoxy phenyl)-6-[1-(2,4-dichlorophenoxy) ethyl]-1,2,4-triazolo[3,4b]-1,3,4-thiadiazole (16c)

Yield: 52\%; m.p: $80{ }^{\circ} \mathrm{C}$; IR $(\mathrm{KBr}) \vee\left(\mathrm{cm}^{-1}\right)$ : 3069 (aromatic $\mathrm{CH}$ stretching), $1613(\mathrm{C}=\mathrm{N}$ stretching), 1587, 1525, 1485, 1451 (C=C ring stretch), 2974, 2865 (methyl $\mathrm{CH}$ stretch), 1261 (asymmetric C-O-C stretching), 1017 (symmetric C-O-C stretching), $1282(\mathrm{~N}-\mathrm{N}=\mathrm{C}) ;{ }^{1} \mathrm{H}$ NMR $\delta$ (ppm): 7.34 (s, 2H, C-2 \& C-6 of Ar), 7.86 (s, 1H, C-3' of Ar), 7.74 (d, 1H, C-5' of Ar), 6.92 (d, $1 \mathrm{H}, \mathrm{C}-6$ ' of Ar'), 5.80 (q, 1H, CH in -CH-CH $\left.{ }_{3}\right), 1.84$ (d, 3H of $\mathrm{CH}_{3}$ in $\left.-\mathrm{CH}-\mathrm{CH}_{3}\right), 3.86(\mathrm{~s}, 9 \mathrm{H}$, $\mathrm{OCH}_{3}$ ); MS m/z: $481 \mathrm{M}^{+}, 483 \mathrm{M}^{+2}, 485 \mathrm{M}^{+4}$; Anal. Calcd. (\%) for $\mathrm{C}_{20} \mathrm{H}_{18} \mathrm{~N}_{4} \mathrm{O}_{4} \mathrm{~S} \mathrm{Cl}_{2}: \mathrm{C}, 49.90$; H, 3.77; N, 11.64; S, 6.66. Found: C, 49.84; H, 3.76; N, 11.63; S, 6.68.

\section{3-(4-pyridinyl)-6-[1-(2,4-dichlorophenoxy)ethyl]-1,2,4-triazolo[3,4-b]-1,3,4-thiadiazole(16d)}

Yield: 54\%; m.p: $148{ }^{\circ} \mathrm{C}$; IR ( $\left.\mathrm{KBr}\right) \vee\left(\mathrm{cm}^{-1}\right): 3075$ (aromatic $\mathrm{CH}$ stretching), $1610(\mathrm{C}=\mathrm{N}$ stretching), 1580, $1565\left(\mathrm{C}=\mathrm{C}\right.$ ring stretch), $1282(\mathrm{~N}-\mathrm{N}=\mathrm{C}) ;{ }^{1} \mathrm{H}$ NMR $\delta(\mathrm{ppm}): 8.04(\mathrm{~d}, 1 \mathrm{H}$, C-3 \& C-5 of Ar), 8.72 (d, 1H, C-2 \& C-6 of Ar), 7.86 (s, 1H, C-3' of Ar), 7.76 (d, 1H, C-5' of $\mathrm{Ar}), 6.94\left(\mathrm{~d}, 1 \mathrm{H}, \mathrm{C}-6\right.$ ' of $\mathrm{Ar}$ '), $5.80\left(\mathrm{q}, 1 \mathrm{H}, \mathrm{CH}\right.$ in $\left.-\mathrm{CH}-\mathrm{CH}_{3}\right), 1.84\left(\mathrm{~d}, 3 \mathrm{H}\right.$ of $\mathrm{CH}_{3}$ in $-\mathrm{CH}-$ $\mathrm{CH}_{3}$ ); MS m/z: $392 \mathrm{M}^{+}, 394 \mathrm{M}^{+2}, 396 \mathrm{M}^{+4}$; Anal. Calcd. (\%) for $\mathrm{C}_{16} \mathrm{H}_{11} \mathrm{~N}_{5} \mathrm{O} \mathrm{S} \mathrm{Cl}_{2}$ : $\mathrm{C}$, 48.99; H, 2.83; N, 17.85; S, 8.17. Found: C, 49.09; H, 2.82; N, 17.78; S, 8.14.

General method for the preparation of 5,6-dihydro-1,2,4-triazolo[3,4-b]-1,3,4thiadiazole $17(a-d)$

An equimolecular mixture of substituted triazole $(0.02 \mathrm{~mol})$ and 2-furfuraldehyde $(0.02$ $\mathrm{mol}), \mathrm{DMF}(30 \mathrm{~mL})$ and a catalytic amount of $p$-toluenesulphonic acid $(10 \mathrm{mg})$ were taken in a round bottom flask. Refluxed the mixture for about 10-12h. The reaction mixture was concentrated to half its volume and cooled to room temperature. Poured the cooled mixture gradually into crushed ice cubes with stirring. Allowed the mixture to stand, solid was separated. It was filtered, washed thoroughly with cold water, dried and recrystallized from hot ethanol. Similarly 18-20 (a-d) were synthesized and characterized.

\section{3-(3,4-dimethoxy phenyl)-6-[2-furanyl]- 5,6-dihydro-1,2,4-triazolo[3,4-b]-1,3,4- thiadiazole (17a)}

Yield: 55(\%); m.p: $172^{\circ} \mathrm{C}$; IR $(\mathrm{KBr}) \vee\left(\mathrm{cm}^{-1}\right): 3370(\mathrm{NH}$ stretching), 3056 (aromatic $\mathrm{CH}$ stretching), 1618 (C=N stretching), 1580, 1535 ( $\mathrm{C}=\mathrm{C}$ ring stretch), 1264 (asymmetric C-O-C stretching), 1017 (symmetric C-O-C stretching); ${ }^{1} \mathrm{H}$ NMR $\delta$ (ppm): 7.1 (d, 1H of C-5 of Ar), 7.5 (s, 1H, C-2 of Ar), 7.68 (d, 1H, C-6 of Ar), 6.84 (d, 1H, C-3' of furan), 7.24 (m, 1H of C-4' of furan), 7.78 (d, 1H C-5' of furan), 5.56 (s, 1H, CH in -CH-NH), 6.20 (s, 1H of NH in -NH-CH), $3.84\left(\mathrm{~s}, 6 \mathrm{H}, \mathrm{OCH}_{3}\right.$ ); MS m/z: $330 \mathrm{M}^{+}$; Anal. Calcd. (\%) for $\mathrm{C}_{15} \mathrm{H}_{14} \mathrm{~N}_{4} \mathrm{O}_{3} \mathrm{~S}: \mathrm{C}$, 54.53; H, 4.27; N, 16.96; S, 9.71. Found: C, 54.46; H, 4.29; N, 16.92; S, 9.74.

\section{3-(3,5-dimethoxy phenyl)-6-[2-furanyl]- 5,6-dihydro-1,2,4-triazolo[3,4-b]-1,3,4- thiadiazole (17b)}

Yield: 52\%; m.p: $160^{\circ} \mathrm{C}$; IR $(\mathrm{KBr}) \vee\left(\mathrm{cm}^{-1}\right)$ : 3350 (NH stretching), 3076 (aromatic $\mathrm{CH}$ stretching), 1617(C=N stretching), 1576, 1552, 1480 (C=C ring stretch), 1262 (asymmetric C-O-C stretching), 1020 (symmetric C-O-C stretching); ${ }^{1} \mathrm{H}$ NMR $\delta$ (ppm): 7.12 (s, 2H, C-2 \& C-6 of Ar), 6.7 (s, 1H, C-4 of Ar), 6.90 (d, 1H, C-3' of furan), 7.24 (m, 1H of C-4' of furan), 7.76 (d, 1H C-5' of furan), 5.58 (s, 1H, CH in CH-NH), 6.18 (s, 1H of NH in -NH-CH), 3.80 (s, 6H, OCH $)$; MS m/z: $330 \mathrm{M}^{+}$; Anal. Calcd. (\%) for $\mathrm{C}_{15} \mathrm{H}_{14} \mathrm{~N}_{4} \mathrm{O}_{3} \mathrm{~S}:$ C, 54.53; H, 4.27; N, 16.96; S, 9.71. Found: C, 54.62; H, 4.29; N, 17.03; S, 9.68. 
3-(3,4,5-trimethoxy phenyl)-6-[2-furanyl]- 5,6-dihydro-1,2,4-triazolo[3,4-b]-1,3,4thiadiazole (17c)

Yield: 59\%; m. p: $172^{\circ} \mathrm{C}$; IR $(\mathrm{KBr}) \vee\left(\mathrm{cm}^{-1}\right): 3328$ (NH stretching), 3080 (aromatic $\mathrm{CH}$ stretching), 1620 (C=N stretching), 1580, 1552, $1484(\mathrm{C}=\mathrm{C}$ ring stretch), 1258 (asymmetric $\mathrm{C}-\mathrm{O}-\mathrm{C}$ stretching), 1023 (symmetric C-O-C stretching); ${ }^{1} \mathrm{H}$ NMR $\delta$ (ppm): (d, 7.32 (s, 2H, C-2 \& C-6 of Ar), 6.90 (d, $1 \mathrm{H}, \mathrm{C}-3$ ' of furan), 7.20 (m, 1H of C-4' of furan), 7.7 (d, 1H C-5' of furan), 5.58 (s, 1H, CH in -CH$\mathrm{NH}), 6.16$ (s, $1 \mathrm{H}$ of $\mathrm{NH}$ in $-\mathrm{NH}-\mathrm{CH}), 3.86\left(\mathrm{~s}, 9 \mathrm{H}, \mathrm{OCH}_{3}\right)$; MS m/z: $360 \mathrm{M}^{+}$; Anal. Calcd. (\%) for $\mathrm{C}_{16} \mathrm{H}_{16} \mathrm{~N}_{4} \mathrm{O}_{4} \mathrm{~S}: \mathrm{C}, 53.32 ; \mathrm{H}, 4.47 ; \mathrm{N}, 15.55 ; \mathrm{S}, 8.90$. Found: C, 53.25; H, 4.48; N, 15.48; S, 8.86.

\section{3-(4-pyridinyl)-6-[2-furanyl]- 5,6-dihydro-1,2,4-triazolo[3,4-b]-1,3,4-thiadiazole (17d)}

Yield: 58\%; m.p: $218^{\circ} \mathrm{C}$; IR ( $\left.\mathrm{KBr}\right) \vee\left(\mathrm{cm}^{-1}\right): 3356(\mathrm{NH}$ stretching), 3082 (aromatic $\mathrm{CH}$ stretching), $1614(\mathrm{C}=\mathrm{N}$ stretching), 1580, 1547 ( $\mathrm{C}=\mathrm{C}$ ring stretch), 1261 (asymmetric $\mathrm{C}-\mathrm{O}-\mathrm{C}$ stretching), 1020 (symmetric C-O-C stretching); ${ }^{1} \mathrm{H}$ NMR $\delta$ (ppm): 8.00 (d, 2H, C-2 \& C-6 of Ar), 8.64 (d, H, C-3 \& C5 of $\mathrm{Ar}$ ), 6.86 (d, 1H, C-3' of furan), 7.26 (m, 1H of C-4' of furan), 7.74 (d, 1H C-5' of furan), 5.58 (s, $1 \mathrm{H}, \mathrm{CH}$ in $-\mathrm{CH}-\mathrm{NH}$ ), 6.20 (s, $1 \mathrm{H}$ of $\mathrm{NH}$ in $-\mathrm{NH}-\mathrm{CH})$; MS $\mathrm{m} / \mathrm{z}: 271 \mathrm{M}^{+}$; Anal. Calcd. (\%) for $\mathrm{C}_{12} \mathrm{H}_{9} \mathrm{~N}_{5} \mathrm{OS}$ : C, 53.13; H, 3.34; N, 25.81; S, 11.82. Found: C, 53.05; H, 3.34; N, 25.74; S, 11.85.

3-(3,4-dimethoxy phenyl)-6-[5-nitro-2-furanyl]- 5,6-dihydro- 1,2,4-triazolo[3,4-b]1,3,4-thiadiazole (18a)

Yield:59\%; m.p: $224^{\circ} \mathrm{C}$; IR (KBr) $\vee\left(\mathrm{cm}^{-1}\right)$ : 3338 (NH stretching), 3086 (aromatic CH stretching), $1619(\mathrm{C}=\mathrm{N}$ stretching), 1590, $1558(\mathrm{C}=\mathrm{C}$ ring stretch), 1265 (asymmetric C-O-C stretching), 1017(symmetric C-O-C stretching), 1527 (asymmetric $\mathrm{NO}_{2}$ stretch), 1347 (symmetric $\mathrm{NO}_{2}$ stretch); ${ }^{1} \mathrm{H}$ NMR $\delta$ (ppm): 7.12 (d, 1H of C-5 of Ar), 7.58 (s,1H, C-2 of Ar), 7.74 (d, 1H, C-6 of Ar), 7.36 (d, 1H, C-3' of furan), 7.90 (d, 1H of C-4' of furan), 5.58 (s, 1H, CH in -CH-NH), 6.20 (s, $1 \mathrm{H}$ of $\mathrm{NH}$ in -NH-CH), $3.82\left(\mathrm{~s}, 6 \mathrm{H}, \mathrm{OCH}_{3}\right)$; $\mathrm{MS} m / z: 375 \mathrm{M}^{+}$; Anal. Calcd. (\%) for $\mathrm{C}_{15} \mathrm{H}_{13} \mathrm{~N}_{5} \mathrm{O}_{5} \mathrm{~S}: \mathrm{C}$, 48.00; H, 3.49; N, 18.66; S, 8.54. Found: C, 48.13; H, 3.51; N, 18.62; S, 8.51.

3-(3,5-dimethoxy phenyl)-6-[5-nitro-2-furanyl]- 5,6-dihydro-1,2,4-triazolo[3,4-b]1,3,4-thiadiazole (18b)

Yield:56\%; m.p: $218^{\circ} \mathrm{C} ; \mathrm{IR}(\mathrm{KBr}) \vee\left(\mathrm{cm}^{-1}\right): 3350$ (NH stretching), 3079 (aromatic $\mathrm{CH}$ stretching), $1615(\mathrm{C}=\mathrm{N}$ stretching), 1584, 1547, 1492 (C=C ring stretch), 1258 (asymmetric C-O-C stretching), 1021 (symmetric C-O-C stretching), 1519 (asymmetric $\mathrm{NO}_{2}$ stretch), 1348 (symmetric $\mathrm{NO}_{2}$ stretch); ${ }^{1} \mathrm{H}$ NMR $\delta$ (ppm): 7.20 (s, 2H, C-2 \& C-6 of Ar), 6.76 (s, 1H, C-4 of Ar), 7.40 (d, 1H, C-3' of furan), 7.94 (d, 1H of C-4' of furan), 5.58 (s, 1H, CH in -CH-NH), 6.16 (s, 1H of NH in -NH-CH), $3.80\left(\mathrm{~s}, 6 \mathrm{H}, \mathrm{OCH}_{3}\right)$; MS m/z: $375 \mathrm{M}^{+}$; Anal. Calcd. (\%) for $\mathrm{C}_{15} \mathrm{H}_{13} \mathrm{~N}_{5} \mathrm{O}_{5} \mathrm{~S}: \mathrm{C}, 48.00 ; \mathrm{H}, 3.49 ; \mathrm{N}$, 18.66; S, 8.54. Found: C, 47.91; H, 3.51; N, 18.72; S, 8.48.

3-(3,4,5-trimethoxy phenyl)-6-[5-nitro-2-furanyl]- 5,6-dihydro-1,2,4-triazolo[3,4b]-1,3,4-thiadiazole (18c)

Yield:60\%; m.p: $214^{\circ} \mathrm{C}$; IR (KBr) $\vee\left(\mathrm{cm}^{-1}\right): 3345$ (NH stretching), 3084 (aromatic CH stretching), $1620 \quad(\mathrm{C}=\mathrm{N}$ stretching), 1580, 1542, $1490 \quad(\mathrm{C}=\mathrm{C}$ ring stretch), 1530 (asymmetric $\mathrm{NO}_{2}$ stretch), 1340 (symmetric $\mathrm{NO}_{2}$ stretch); ${ }^{1} \mathrm{H}$ NMR $\delta$ (ppm): 7.28 (s, 2H, C-2 \& C-6 of Ar), 7.42 (d, 1H, C-3' of furan), 7.92 (d, 1H of C-4' of furan), 5.60 (s, 1H, $\mathrm{CH}$ in -CH-NH), 6.18 (s, $1 \mathrm{H}$ of $\mathrm{NH}$ in $-\mathrm{NH}-\mathrm{CH}), 3.84\left(\mathrm{~s}, 9 \mathrm{H}, \mathrm{OCH}_{3}\right) ; \mathrm{MS} \mathrm{m} / z: 405 \mathrm{M}^{+}$; Anal. Calcd. (\%) for $\mathrm{C}_{16} \mathrm{H}_{15} \mathrm{~N}_{5} \mathrm{O}_{6} \mathrm{~S}: \mathrm{C}, 47.40 ; \mathrm{H}, 3.73 ; \mathrm{N}, 17.28 ; \mathrm{S}, 7.91$. Found: $\mathrm{C}$, 47.41; H, 3.76; N, 17.24; S, 7.87. 
3-(4-pyridinyl)-6-[5-nitro-2-furanyl]- 5,6-dihydro-1,2,4-triazolo[3,4-b]-1,3,4thiadiazole (18d)

Yield: 57\%; m.p: $234^{\circ} \mathrm{C}$; IR (KBr) v $\left(\mathrm{cm}^{-1}\right)$ : 3327 (NH stretching), 3068 (aromatic $\mathrm{CH}$ stretching), $1620\left(\mathrm{C}=\mathrm{N}\right.$ stretching), 1590, $1560\left(\mathrm{C}=\mathrm{C}\right.$ ring stretch), 1526 (asymmetric $\mathrm{NO}_{2}$ stretch), 1342 (symmetric $\mathrm{NO}_{2}$ stretch); ${ }^{1} \mathrm{H}$ NMR $\delta$ (ppm): 8.00 (d, 2H, C-2 \& C-6 of Ar), 8.64 (d, H, C-3 \& C-5 of Ar), 7.40 (d, 1H, C-3' of furan), 7.92 (d, 1H of C-4' of furan), 5.58 (s, 1H, CH in -CH-NH), 6.16 (s, $1 \mathrm{H}$ of NHin -NH-CH); MS $m / z: 316 \mathrm{M}^{+}$; Anal. Calcd. (\%) for $\mathrm{C}_{12} \mathrm{H}_{8} \mathrm{~N}_{6} \mathrm{O}_{3} \mathrm{~S}: \mathrm{C}, 45.57$; H, 2.55; N, 26.57; S, 10.14. Found: C, 45.48; H, 2.55; N, 26.62; S, 10.11.

3-(3,4-dimethoxy phenyl)-6-[pyrol-2-yl]- 5,6-dihydro-1,2,4-triazolo[3,4-b]-1,3,4thiadiazole (19a)

Yield: 53\%; m.p: $176{ }^{\circ} \mathrm{C}$; IR ( $\left.\mathrm{KBr}\right) \vee\left(\mathrm{cm}^{-1}\right)$ : 3352 (NH stretching), 3070 (aromatic $\mathrm{CH}$ stretching), 1619 ( $\mathrm{C}=\mathrm{N}$ stretching), 1586, 1554 ( $\mathrm{C}=\mathrm{C}$ ring stretch), 1263 (asymmetric $\mathrm{C}-\mathrm{O}-\mathrm{C}$ stretching), 1019 (symmetric C-O-C stretching); ${ }^{1} \mathrm{H}$ NMR $\delta$ (ppm): 7.20 (d, 1H of C-5 of Ar), 7.58 (s,1H, C-2 of Ar), 7.76 (d, 1H, C-6 of Ar), 6.42 (d, 1H, C-3' of pyrrole), 6.60 (m, 1H of C-4' of pyrrole), 7.04 (d, 1H of C-5' of pyrrole), 5.60 (s, 1H, CH in -CH-NH), 6.16 (s, 1H of NH in -NH-CH), 9.8 (d, 1H, -NH of pyrrole), 3.80 (s, $6 \mathrm{H}, \mathrm{OCH}_{3}$ ); MS m/z: $329 \mathrm{M}^{+}$; Anal. Calcd. (\%) for $\mathrm{C}_{15} \mathrm{H}_{15} \mathrm{~N}_{5} \mathrm{O}_{2} \mathrm{~S}: \mathrm{C}, 54.70 ; \mathrm{H}$, 4.59; N, 21.26; S, 9.74. Found: C, 54.62; H, 4.61; N, 21.18; S, 9.71.

3-(3,5-dimethoxy phenyl)-6-[pyrol-2-yl]- 5,6-dihydro-1,2,4-triazolo [3,4-b]-1,3,4thiadiazole (19b)

Yield: $50 \%$; m.p: $162^{\circ} \mathrm{C}$; IR $(\mathrm{KBr}) \vee\left(\mathrm{cm}^{-1}\right): 3365$ (NH stretching), 3083 (aromatic $\mathrm{CH}$ stretching), 1617(C=N stretching), 1586, 1550, 1470 (C=C ring stretch), 1257 (asymmetric C-OC stretching), 1021 (symmetric C-O-C stretching); ${ }^{1} \mathrm{H}$ NMR $\delta$ (ppm): 7.26 (s, 2H, C-2 \& C-6 of $\mathrm{Ar}), 6.80$ (s, 1H, C-4 of Ar), 6.46 (d, 1H, C-3' of pyrrole), 6.60 (m, 1H of C-4' of pyrrole), 7.06 (d, $1 \mathrm{H}$ of C-5' of pyrrole), 5.60 (s, 1H, CH in -CH-NH), 6.16 (s, 1H of NH in -NH-CH), 9.6 (d, $1 \mathrm{H}$, -NH of pyrrole), 3.80 (s, $6 \mathrm{H}, \mathrm{OCH}_{3}$ ); $\mathrm{MS} m / z$ : $329 \mathrm{M}^{+}$; Anal. Calcd. (\%) for $\mathrm{C}_{15} \mathrm{H}_{15} \mathrm{~N}_{5} \mathrm{O}_{2} \mathrm{~S}$ : C, 54.70; H, 4.59; N, 21.26; S, 9.74. Found: C, 54.63; H, 4.60; N, 21.32; S, 9.71.

3-(3,4,5-trimethoxy phenyl)-6-[pyrol-2-yl]- 5,6-dihydro- 1,2,4-triazolo[3,4-b]-1,3,4thiadiazole (19c)

Yield: 55\%; m.p: $180^{\circ} \mathrm{C}$; IR $(\mathrm{KBr}) \vee\left(\mathrm{cm}^{-1}\right): 3348$ (NH stretching), 3076 (aromatic $\mathrm{CH}$ stretching), 1615 ( $\mathrm{C}=\mathrm{N}$ stretching), 1580, 1568, 1490 (C=C ring stretch), 1259 (asymmetric C-OC stretching), 1024 (symmetric C-O-C stretching); ${ }^{1} \mathrm{H}$ NMR $\delta$ (ppm): ${ }^{1} \mathrm{H}$ NMR $\delta$ (ppm): 7.34 (s, 2H, C-2 \& C-6 of Ar), 6.42 (d, 1H, C-3' of pyrrole), 6.59 (m, 1H of C-4' of pyrrole), 7.06 (d, $1 \mathrm{H}$ of C-5' of pyrrole), 5.60 (s, 1H, CH in -CH-NH), 6.18 (s, 1H of NH in -NH-CH), 9.8 (d, 1H, -NH of pyrrole), 3.84 (s, $9 \mathrm{H}, \mathrm{OCH}_{3}$ ); $\mathrm{MS} m / z: 359 \mathrm{M}^{+}$; Anal. Calcd. (\%) for $\mathrm{C}_{16} \mathrm{H}_{17} \mathrm{~N}_{5} \mathrm{O}_{3} \mathrm{~S}: \mathrm{C}, 53.47$; H, 4.77; N, 19.49; S, 8.92. Found: C, 53.45; H, 4.77; N, 19.42; S, 8.87.

\section{3-(4-pyridinyl)-6-[pyrol-2-yl]- 5,6-dihydro-1,2,4-triazolo[3,4-b]-1,3,4-thiadiazole (19d)}

Yield: 53\%; m. p: $206^{\circ} \mathrm{C}$; IR (KBr) $\vee\left(\mathrm{cm}^{-1}\right): 3365$ (NH stretching), 3086 (aromatic CH stretching), $1618(\mathrm{C}=\mathrm{N}$ stretching $), 1588,1566\left(\mathrm{C}=\mathrm{C}\right.$ ring stretch); ${ }^{1} \mathrm{H}$ NMR $\delta(\mathrm{ppm}): 7.96(\mathrm{~d}, 2 \mathrm{H}, \mathrm{C}-2 \& \mathrm{C}-6$ of Ar), 8.68 (d, H, C-3 \& C-5 of Ar), 6.40 (d, 1H, C-3' of pyrrole), 6.62 (m, 1H of C-4' of pyrrole), 7.04 (d, 1H of C-5' of pyrrole), 5.60 (s, 1H, CH in -CH-NH), 6.18 (s, 1H of NH in -NH-CH), 9.8 (d, $1 \mathrm{H}$, -NH of pyrrole); MS $m / z: 270 \mathrm{M}^{+}$; Anal. Calcd. (\%) for $\mathrm{C}_{12} \mathrm{H}_{10} \mathrm{~N}_{6} \mathrm{~S}: \mathrm{C}, 53.32 ; \mathrm{H}, 3.73 ; \mathrm{N}$, 31.09; S, 11.86. Found: C, 53.25; H, 3.73; N, 31.14; S, 11.80. 


\section{3-(3,4-dimethoxy phenyl)-6-[N-methyl-pyrol-2-yl]-1,2,4-triazolo[3,4-b]-1,3,4-} thiadiazole (20a)

Yield: 57(\%); m.p: $184^{\circ} \mathrm{C}$; IR ( $\left.\mathrm{KBr}\right) \vee\left(\mathrm{cm}^{-1}\right)$ : 3364 (NH stretching), 3062 (aromatic $\mathrm{CH}$ stretching), $1610(\mathrm{C}=\mathrm{N}$ stretching), 1580, 1553 ( $\mathrm{C}=\mathrm{C}$ ring stretch), 1266 (asymmetric $\mathrm{C}-\mathrm{O}-\mathrm{C}$ stretching), 1021 (symmetric C-O-C stretching), 2963, 2847 (methyl CH stretch); ${ }^{1} \mathrm{H}$ NMR $\delta$ (ppm): 7.24 (d, 1H of C-5 of Ar), 7.56 (s,1H, C-2 of Ar), 7.72 (d, 1H, C-6 of Ar), 6.88 (d, 1H, C-5' of pyrrole), 6.56 (m, $1 \mathrm{H}, \mathrm{C}-4$ ' of pyrrole), 6.40 (d, 1H, C-3' of pyrrole), 5.62 (s, 1H, of -CH-NH), 6.20 (s, 1H of NH in $\mathrm{NH}-\mathrm{CH}), 3.56\left(\mathrm{~s}, 3 \mathrm{H}\right.$ of $\left.\mathrm{N}_{-} \mathrm{CH}_{3}\right), 3.82\left(\mathrm{~s}, 6 \mathrm{H}, \mathrm{OCH}_{3}\right)$; MS m/z: $343 \mathrm{M}^{+}$; Anal. Calcd. (\%) for $\mathrm{C}_{16}$ $\mathrm{H}_{17} \mathrm{~N}_{5} \mathrm{O}_{2} \mathrm{~S}$ : C, 55.96; H, 4.99; N, 20.39; S, 9.34. Found: C, 56.10; H, 5.01; N, 20.31; S, 9.31.

\section{3-(3,5-dimethoxyphenyl)-6-[N-methyl-pyrol-2-yl]-1,2,4-triazolo[3,4-b]-1,3,4-thiadiazole(20b)}

Yield: 52\%; m.p: $174^{\circ} \mathrm{C}$; $\mathrm{R}(\mathrm{KBr}) \vee\left(\mathrm{cm}^{-1}\right): 3364$ (NH stretching), 3058 (aromatic $\mathrm{CH}$ stretching), $1615(\mathrm{C}=\mathrm{N}$ stretching), 1589, 1565, 1480 ( $\mathrm{C}=\mathrm{C}$ ring stretch), 1258 (asymmetric C-O-C stretching), 1025 (symmetric C-O-C stretching), 2958, 2849 (methyl CH stretch); ${ }^{1} \mathrm{H}$ NMR $\delta$ (ppm): 7.22 (s, 2H, C-2 \& C-6 of Ar), 6.76 (s, 1H, C-4 of Ar), 6.9 (d, 1H, C-5' of pyrrole), 6.52 (m, 1H, C-4' of pyrrole), 6.38 (d, 1H, C-3' of pyrrole), 5.48 (s, 1H, of -CH-NH), 6.18 (s, 1H of NH in -NH-CH), $3.60\left(\mathrm{~s}, 3 \mathrm{H}\right.$ of $\mathrm{N}_{-} \mathrm{CH}_{3}$ ), $3.82\left(\mathrm{~s}, 6 \mathrm{H}, \mathrm{OCH}_{3}\right)$; MS m/z: $343 \mathrm{M}^{+}$; Anal. Calcd. (\%) for $\mathrm{C}_{16} \mathrm{H}_{17} \mathrm{~N}_{5} \mathrm{O}_{2} \mathrm{~S}$ : C, 55.96; H, 4.99; N, 20.39; S, 9.34. Found: C, 56.05; H, 5.01; N, 20.34; S, 9.32.

\section{3-(3,4,5-trimethoxyphenyl)-6-[N-methyl-pyrol-2-yl]-1,2,4-triazolo[3,4-b]-1,3,4- thiadiazole (20c)}

Yield: $61 \%$; m. p: $162^{\circ} \mathrm{C}$; IR $(\mathrm{KBr}) \vee\left(\mathrm{cm}^{-1}\right): 3345$ (NH stretching), 3072 (aromatic $\mathrm{CH}$ stretching), 1616 ( $\mathrm{C}=\mathrm{N}$ stretching), 1587, 1549, $1480(\mathrm{C}=\mathrm{C}$ ring stretch), 1264 (asymmetric $\mathrm{C}-\mathrm{O}-\mathrm{C}$ stretching), 1022 (symmetric C-O-C stretching), 2965, 2850 (methyl CH stretch); ${ }^{1} \mathrm{H}$ NMR $\delta$ (ppm): 7.3 (s, 2H, C-2 \& C-6 of Ar), 7.06 (d, 1H, C-5' of pyrrole), 6.82 (m, 1H, C-4' of pyrrole), 6.64 (d, 1H, C-3' of pyrrole), $5.50(\mathrm{~s}, 1 \mathrm{H}$, of $-\mathrm{CH}-\mathrm{NH}), 6.16(\mathrm{~s}, 1 \mathrm{H}$ of $\mathrm{NH}$ in $-\mathrm{NH}-\mathrm{CH}), 3.60\left(\mathrm{~s}, 3 \mathrm{H}\right.$ of $\left.\mathrm{N}_{-} \mathrm{CH}_{3}\right), 3.86$ (s, $9 \mathrm{H}, \mathrm{OCH}_{3}$ ); MS m/z: $373 \mathrm{M}^{+}$; Anal. Calcd. (\%) for $\mathrm{C}_{17} \mathrm{H}_{19} \mathrm{~N}_{5} \mathrm{O}_{3} \mathrm{~S}: \mathrm{C}, 54.68 ; \mathrm{H}, 5.13 ; \mathrm{N}, 18.75 ; \mathrm{S}$, 8.59. Found: C, 54.61; H, 5.13; N, 18.67; S, 8.61.

\section{3-(4-pyridinyl)-6-[N-methyl-pyrol-2-yl]-5,6-dihydro-1,2,4-triazolo[3,4-b]-1,3,4- thiadiazole (20d)}

Yield: 59\%; m. p: $200^{\circ} \mathrm{C}$; IR ( $\left.\mathrm{KBr}\right) \vee\left(\mathrm{cm}^{-1}\right): 3369$ (NH stretching), 3045 (aromatic $\mathrm{CH}$ stretching), 1614 (C=N stretching), 1590, 1563 (C=C ring stretch), 2964, 2840 (methyl CH stretch); ${ }^{1} \mathrm{H}$ NMR $\delta$ (ppm): 8.02 (d, 2H, C-2 \& C-6 of Ar), 8.66 (d, H, C-3 \& C-5 of Ar), 6.86 (d, 1H, C-5' of pyrrole), $6.56(\mathrm{~m}, 1 \mathrm{H}, \mathrm{C}-4$ ' of pyrrole), 6.38 (d, 1H, C-3' of pyrrole), 5.48 (s, 1H, of -CH-NH), $6.16(\mathrm{~s}, 1 \mathrm{H}$ of NH in -NH-CH), 3.50 (s, $3 \mathrm{H}$ of $\mathrm{N}_{-} \mathrm{CH}_{3}$ ), MS $m / z: 284 \mathrm{M}^{+}$; Anal. Calcd. (\%) for $\mathrm{C}_{13} \mathrm{H}_{12} \mathrm{~N}_{6} \mathrm{~S}: \mathrm{C}$, 54.91; H, 4.25; N, 29.56; S, 11.28. Found: C, 54.81; H, 4.22; N, 29.52; S, 11.25.

\section{Results and Discussion}

In the IR spectra of the cyclized products showed absorption band at $3270-3290 \mathrm{~cm}^{-1}$ due to $\mathrm{NH}$ functional group and the weak broad absorption band around $2580 \mathrm{~cm}^{-1}$ due to $\mathrm{SH}$ group were absent. This confirmed the involvement of $\mathrm{NH}_{2}$ and $\mathrm{SH}$ groups of the parent amino mercapto triazole in the ring formation. An absorption band was observed for all the synthesized compounds in the range of $3060-3090 \mathrm{~cm}^{-1}$ may be attributed for aromatic stretching vibration, while that seen at $1610-1614 \mathrm{~cm}^{-1}$ corresponds to $\mathrm{C}=\mathrm{N}$ linkage. Thus, the formation of iminomethine functional group in the compound was indicated. 
In the ${ }^{1} \mathrm{H}$ NMR spectra of synthesized compound, the peaks due to $\mathrm{NH}_{2}$ and $\mathrm{SH}$, which were present in the amino mercapto triazole were absent that further confirmed the involvement of these functional groups in the cyclization of triazole to triazolo thiadiazoles. Similarly the absence of $\mathrm{SH}$ proton and the down fielding of $\mathrm{NH}_{2}$ protons (integration for one proton) in the ${ }^{1} \mathrm{H}$ NMR spectra established that - $\mathrm{CHO}$ group of the aromatic aldehydes reacted with $-\mathrm{SH} \&-\mathrm{NH}_{2}$ groups of triazoles and thus converted to dihydro triazolo thiadiazoles (17-20). The ${ }^{1} \mathrm{H}$ NMR, mass spectra, IR and elemental analysis supported the structure of various synthesized triazolo thiadiazoles and their dihydro analogues.

The antimicrobial results showed that some of the compounds are active against the tested microbes. It can be concluded that none of the prepared compounds were superior to positive controls against various tested microbial strains, but was interesting to note that compounds $8 \mathrm{~d}, 9 \mathrm{a}, 9 \mathrm{~b}, 9 \mathrm{~d}, 14 \mathrm{~d}$ \& 16d were very sensitive to all the tested organisms comparable to the standards used at the concentration of $30 \mu \mathrm{g} / \mathrm{mL}$. Antimicrobial effects of the some of the synthesized compounds were reported in Table $3 \& 4$ as zone of inhibition against various bacterial and fungal strains respectively.

Anti-inflammatory and analgesic activity screening indicated that some of the tested compound 10d, 12d, 14a, 14b, 14d, 16a, 16b and 16d showed good anti-inflammatory and analgesic activities. Other compounds had moderate anti-inflammatory and analgesic activities. Anti-inflammatory and analgesic effects of the newly synthesized compounds were reported in Table $1 \& 2$ respectively.

Table 1. Anti-inflammatory activity of compounds

Compd Change in paw volume (in $\mathrm{mL}$ ) after $( \pm \mathrm{SE})^{\#} \quad$ Percentage inhibition of oedema

\begin{tabular}{|c|c|c|c|c|c|c|c|c|}
\hline & $1 \mathrm{~h}$ & $2 \mathrm{~h}$ & $3 \mathrm{~h}$ & $4 \mathrm{~h}$ & $1 \mathrm{~h}$ & $2 \mathrm{~h}$ & $3 \mathrm{~h}$ & $4 \mathrm{~h}$ \\
\hline $8 \mathrm{a}$ & $0.71 \pm 0.02$ & $0.98 \pm 0.03$ & $1.39 \pm 0.02$ & $1.52 \pm 0.02$ & 11.4 & 16.2 & 20.7 & 26.5 \\
\hline $8 b$ & $0.73 \pm 0.02$ & $1.02 \pm 0.04$ & $1.41 \pm 0.03$ & $1.56 \pm 0.03$ & 8.8 & 12.9 & 19.5 & 24.6 \\
\hline $8 \mathrm{c}$ & $0.74 \pm 0.02$ & $1.00 \pm 0.06$ & $1.45 \pm 0.04$ & $1.64 \pm 0.03$ & 7.8 & 13.9 & 17.4 & 20.5 \\
\hline $8 \mathrm{~d}$ & $0.71 \pm 0.02$ & $0.97 \pm 0.05$ & $1.36 \pm 0.02$ & $1.52 \pm 0.02$ & 11.3 & 16.8 & 22.3 & 26.4 \\
\hline $10 \mathrm{a}$ & $0.80 \pm 0.02$ & $1.07 \pm 0.07$ & $1.43 \pm 0.02$ & $1.66 \pm 0.02$ & 11.2 & 16.5 & 20.7 & 26.4 \\
\hline $10 \mathrm{~b}$ & $0.79 \pm 0.05$ & $1.07 \pm 0.03$ & $1.42 \pm 0.03$ & $1.65 \pm 0.04$ & 12.3 & 16.9 & 21.1 & 26.8 \\
\hline $10 \mathrm{c}$ & $0.82 \pm 0.05$ & $1.10 \pm 0.07$ & $1.49 \pm 0.02$ & $1.77 \pm 0.03$ & 8.6 & 14.0 & 17.1 & 21.5 \\
\hline $10 \mathrm{~d}$ & $0.77 \pm 0.02$ & $1.02 \pm 0.02$ & $1.32 \pm 0.03$ & $1.53 \pm 0.04$ & $14.9^{*}$ & $20.5^{*}$ & $26.7^{*}$ & $31.9^{*}$ \\
\hline $12 \mathrm{a}$ & $0.76 \pm 0.02$ & $1.00 \pm 0.02$ & $1.32 \pm 0.03$ & $1.58 \pm 0.07$ & $15.6^{*}$ & $22.1^{*}$ & $26.8^{*}$ & $29.8^{*}$ \\
\hline $12 b$ & $0.77 \pm 0.04$ & $1.01 \pm 0.03$ & $1.32 \pm 0.04$ & $1.61 \pm 0.05$ & $14.7^{*}$ & $21.2^{*}$ & $26.4^{*}$ & $28.5^{*}$ \\
\hline $12 c$ & $0.78 \pm 0.04$ & $1.06 \pm 0.02$ & $1.39 \pm 0.03$ & $1.70 \pm 0.03$ & $13.3^{*}$ & 17.1 & 22.8 & 24.4 \\
\hline $12 d$ & $0.74 \pm 0.03$ & $0.93 \pm 0.02$ & $1.19 \pm 0.05$ & $1.44 \pm 0.02$ & 17.8 & 27.9 & $33.9^{* * *}$ & $36.2^{* *}$ \\
\hline $14 \mathrm{a}$ & $0.61 \pm 0.02$ & $0.83 \pm 0.03$ & $1.08 \pm 0.03$ & $1.33 \pm 0.03$ & $17.4^{* *}$ & $23.5^{*}$ & $29.6^{*}$ & $32.8^{*}$ \\
\hline $14 b$ & $0.60 \pm 0.03$ & $0.83 \pm 0.02$ & $1.10 \pm 0.07$ & $1.36 \pm 0.05$ & $17.8^{* *}$ & $23.2^{*}$ & $27.9^{*}$ & $31.6^{*}$ \\
\hline $14 c$ & $0.62 \pm 0.03$ & $0.89 \pm 0.03$ & $1.17 \pm 0.05$ & $1.46 \pm 0.023$ & $15.3^{*}$ & 18.3 & 23.8 & 26.4 \\
\hline $14 d$ & $0.58 \pm 0.02$ & $0.79 \pm 0.06$ & $1.04 \pm 0.02$ & $1.26 \pm 0.02$ & $20.3^{*}$ & $26.7^{* *}$ & $32.3^{* * *}$ & $36.6^{* *}$ \\
\hline $16 a$ & $0.62 \pm 0.02$ & $0.85 \pm 0.05$ & $1.09 \pm 0.02$ & $1.35 \pm 0.04$ & $15.9^{* *}$ & $21.7^{*}$ & $29.0^{*}$ & $32.1^{*}$ \\
\hline $16 b$ & $0.61 \pm 0.02$ & $0.82 \pm 0.04$ & $1.07 \pm 0.02$ & $1.32 \pm 0.02$ & $16.9^{* * *}$ & $24.7^{* *}$ & $29.9^{*}$ & $33.2^{*}$ \\
\hline $16 c$ & $0.63 \pm 0.04$ & $0.86 \pm 0.02$ & $1.16 \pm 0.02$ & $1.45 \pm 0.02$ & $14.4^{*}$ & $20.6^{*}$ & 24.0 & 26.8 \\
\hline $16 \mathrm{~d}$ & $0.55 \pm 0.03$ & $0.82 \pm 0.02$ & $1.07 \pm 0.05$ & $1.30 \pm 0.04$ & $18.9^{*}$ & $24.3^{* *}$ & $30.1^{*}$ & $34.6^{* *}$ \\
\hline $\begin{array}{l}\text { Phenyl } \\
\text { butazon }\end{array}$ & $0.72 \pm 0.03$ & $0.92 \pm 0.03$ & $0.97 \pm 0.04$ & $1.00 \pm 0.00$ & $21.2^{* *}$ & $33.5^{* * *}$ & $40.8^{* * *}$ & $* 48.0^{* * *}$ \\
\hline
\end{tabular}

$\#= \pm$ Standard error $; *=\mathrm{P}<0.05 ; * *=\mathrm{P}<0.01 ; * * *=\mathrm{P}<0.001 ;$ For all other comparison $\mathrm{P}>0.05$. 
Table 2. Analgesic activities of compounds

\begin{tabular}{|c|c|c|c|c|c|c|}
\hline \multirow[t]{2}{*}{ Compd } & \multicolumn{3}{|c|}{ Reaction time (sec) after drug administration } & \multicolumn{3}{|c|}{ Percent increase in reaction time } \\
\hline & $30 \mathrm{~min} \pm \mathrm{SE}^{\#}$ & $60 \min \pm \mathrm{SE}^{\#}$ & $90 \min \pm \mathrm{SE}^{\#}$ & $30 \mathrm{~min}$ & $60 \mathrm{~min}$ & $90 \mathrm{~min}$ \\
\hline $8 \mathrm{~d}$ & $5.98 \pm 0.43$ & $6.30 \pm 0.43$ & $6.64 \pm 0.21$ & 19.2 & $23.3^{*}$ & $27.3^{*}$ \\
\hline $9 d$ & $7.81 \pm 0.31$ & $8.37 \pm 0.34$ & $8.68 \pm 0.45$ & 19.0 & $24.4^{*}$ & $27.1^{*}$ \\
\hline $10 \mathrm{~d}$ & $8.23 \pm 0.33$ & $8.84 \pm 0.55$ & $9.29 \pm 0.45$ & 19.1 & $24.7^{*}$ & $28.3^{*}$ \\
\hline $12 \mathrm{a}$ & $8.70 \pm 0.73$ & $9.27 \pm 0.43$ & $9.73 \pm 0.35$ & 17.7 & 22.8 & $26.4^{*}$ \\
\hline $12 b$ & $7.23 \pm 0.24$ & $8.02 \pm 0.45$ & $8.46 \pm 0.34$ & 14.8 & $23.2^{*}$ & $27.2^{*}$ \\
\hline $12 c$ & $7.47 \pm 0.56$ & $7.77 \pm 0.50$ & $8.63 \pm 0.61$ & 11.6 & 19.1 & 23.5 \\
\hline $12 d$ & $3.98 \pm 0.33$ & $4.29 \pm 0.60$ & $4.57 \pm 0.31$ & 20.5 & $26.2^{* *}$ & $30.7^{*}$ \\
\hline $14 \mathrm{a}$ & $8.34 \pm 0.34$ & $9.20 \pm 0.21$ & $10.03 \pm 0.40$ & 20.1 & $27.6^{* * *}$ & $33.6^{* *}$ \\
\hline $14 b$ & $8.54 \pm 0.52$ & $9.08 \pm 0.45$ & $9.48 \pm 0.60$ & 23.9 & $28.4^{* *}$ & $31.4^{* *}$ \\
\hline $14 \mathrm{c}$ & $9.53 \pm 0.34$ & $10.16 \pm 0.42$ & $10.52 \pm 0.34$ & 21.3 & $26.2^{* *}$ & $28.7^{*}$ \\
\hline $14 d$ & $8.39 \pm 0.15$ & $9.21 \pm 0.42$ & $10.09 \pm 0.45$ & 26.6 & $33.1^{* * * *}$ & $38.9^{* *}$ \\
\hline $15 d$ & $4.44 \pm 0.47$ & $4.75 \pm 0.56$ & $5.06 \pm 0.37$ & 17.6 & 22.9 & 27.7 \\
\hline $16 a$ & $5.60 \pm 0.15$ & $6.26 \pm 0.42$ & $6.63 \pm 0.45$ & 22.7 & $30.8^{* *}$ & $34.7^{* *}$ \\
\hline $16 b$ & $9.60 \pm 0.33$ & $10.69 \pm 0.48$ & $11.42 \pm 0.17$ & 20.2 & $28.3^{*}$ & $32.9^{* *}$ \\
\hline $16 \mathrm{c}$ & $7.19 \pm 0.34$ & $8.13 \pm 0.37$ & $8.47 \pm 0.21$ & 16.6 & $26.2^{*}$ & $29.2^{*}$ \\
\hline $16 \mathrm{~d}$ & $5.91 \pm 0.34$ & $6.89 \pm 0.42$ & $7.28 \pm 0.34$ & 23.9 & $34.7^{* * * *}$ & $38.2^{* *}$ \\
\hline $17 d$ & $6.24 \pm 0.17$ & $6.92 \pm 0.21$ & $7.84 \pm 0.40$ & 5.68 & 15.18 & 26.14 \\
\hline $18 \mathrm{~d}$ & $6.17 \pm 0.34$ & $6.85 \pm 0.56$ & $8.70 \pm 0.37$ & 5.34 & 15.68 & 26.85 \\
\hline $\begin{array}{l}\text { diclofena } \\
\text { sodium }\end{array}$ & ac $4.74 \pm 0.50$ & $5.58 \pm 0.43$ & $7.33 \pm 0.21$ & $26.2^{* * *}$ & $37.3^{* * *}$ & $52.3^{* * * *}$ \\
\hline
\end{tabular}

\section{Pharmacology}

\section{Anti-inflammatory activity}

All the synthesized compounds were evaluated for their anti-inflammatory activity against carrageenan-induced acute paw oedema in albino rats (Wistar strain) weighing 150$200 \mathrm{~g}^{15,16}$. The animals were weighed and numbered into various groups and each group contained six animals. One group served as control and received $0.1 \mathrm{~mL}$ of $1 \%$ gum acacia suspension orally. Group II served as standard and received phenylbutazone at a dose of $5 \mathrm{mg} / \mathrm{kg}$ suspension in gum acacia orally. One hour after the administration of test compounds at a dose of $5 \mathrm{mg} / \mathrm{kg}$ as suspension in gum acacia, $0.1 \mathrm{~mL}$ of $1 \%$ carrageenan in normal saline was given subcutaneously to the sub plantar region of right hind paw. The paw volume was measured immediately ( ' 0 ' $\mathrm{h}$ ) and after $1,2,3$ and $4^{\text {th }} \mathrm{h}$ respectively by using plethysmograph. The amount of oedema in the drug-treated groups was compared in relation to the control group with the corresponding time intervals. The percentage of inhibition by the drugs was calculated by using the formula,

Percentage inhibition $=100\left(1-\mathrm{V}_{\text {test }} / \mathrm{V}_{\text {control }}\right)$,

Where $\mathrm{V}_{\text {test }}=$ mean increase in paw volume of drug treated group;

$\mathrm{V}_{\text {control }}=$ mean increase in paw volume of control group.

The results were expressed as percentage inhibition of oedema over the untreated control group. 
Table 3. Antifungal activities of compounds

\begin{tabular}{|c|c|c|c|c|c|c|}
\hline \multirow[t]{2}{*}{ Compd } & \multicolumn{3}{|c|}{ C. albicans ${ }^{1}$} & \multicolumn{3}{|c|}{ A. niger ${ }^{2}$} \\
\hline & $\begin{array}{c}10, \mu \mathrm{g} / \mathrm{mL} \\
\pm \mathrm{SD}^{*}\end{array}$ & $\begin{array}{c}20, \mu \mathrm{g} / \mathrm{mL} \\
\pm \mathrm{SD}^{*}\end{array}$ & $\begin{array}{c}30, \underset{ \pm S D}{\mu} / \mathrm{mL} \\
\pm \mathrm{SD}^{*}\end{array}$ & $\begin{array}{l}10, \mu \mathrm{g} / \mathrm{mL} \\
\pm \mathrm{SD}^{*}\end{array}$ & $\begin{array}{l}20, \mu \mathrm{g} / \mathrm{mL} \\
\pm \mathrm{SD}^{*}\end{array}$ & $\begin{array}{l}30, \mu \mathrm{g} / \mathrm{mL} \\
\pm \mathrm{SD}^{*}\end{array}$ \\
\hline $4 a$ & $5.64 \pm 1.16$ & $12.84 \pm 1.73$ & $18.78 \pm 1.53$ & $6.04 \pm 0.58$ & $11.63 \pm 0.58$ & $17.58 \pm 1.16$ \\
\hline $4 \mathrm{~b}$ & $5.82 \pm 0.58$ & $11.37 \pm 0.58$ & $17.95 \pm 1.73$ & $6.14 \pm 1.16$ & $11.54 \pm 1.53$ & $16.72 \pm 0.58$ \\
\hline $4 d$ & $6.27 \pm 0.58$ & $14.84 \pm 1.16$ & $19.36 \pm 1.53$ & $5.92 \pm 0.58$ & $11.00 \pm 0.00$ & $17.67 \pm 0.58$ \\
\hline $5 a$ & $6.18 \pm 1.16$ & $13.82 \pm 0.58$ & $18.75 \pm 0.61$ & $5.62 \pm 0.47$ & $12.62 \pm 0.58$ & $18.54 \pm 0.27$ \\
\hline $5 b$ & $5.84 \pm 0.53$ & $12.62 \pm 0.58$ & $19.00 \pm 2.00$ & $6.00 \pm 1.00$ & $13.46 \pm 0.58$ & $17.42 \pm 1.16$ \\
\hline $5 d$ & $5.48 \pm 0.58$ & $12.00 \pm 1.00$ & $17.48 \pm 1.53$ & $6.25 \pm 0.58$ & $13.00 \pm 1.00$ & $16.72 \pm 0.27$ \\
\hline $6 a$ & $6.48 \pm 0.58$ & $13.92 \pm 1.73$ & $18.00 \pm 1.00$ & $6.40 \pm 0.61$ & $12.85 \pm 0.58$ & $19.10 \pm 1.53$ \\
\hline $6 b$ & $6.14 \pm 0.58$ & $13.66 \pm 0.47$ & $17.82 \pm 0.27$ & $6.32 \pm 0.61$ & $13.00 \pm 1.00$ & $19.00 \pm 0.00$ \\
\hline $6 d$ & $5.78 \pm 0.58$ & $11.00 \pm 1.00$ & $19.24 \pm 1.53$ & $6.14 \pm 0.58$ & $12.00 \pm 1.00$ & $19.10 \pm 0.58$ \\
\hline $7 d$ & $5.34 \pm 0.58$ & $10.00 \pm 2.00$ & $17.28 \pm 1.53$ & $5.14 \pm 0.58$ & $9.48 \pm 0.58$ & $16.84 \pm 0.58$ \\
\hline $8 a$ & $7.14 \pm 0.47$ & $13.72 \pm 0.58$ & $19.28 \pm 0.47$ & $6.64 \pm 0.58$ & $11.84 \pm 1.73$ & $18.42 \pm 1.16$ \\
\hline $8 b$ & $6.82 \pm 0.58$ & $12.88 \pm 0.61$ & $19.36 \pm 0.61$ & $7.23 \pm 0.58$ & $13.00 \pm 0.00$ & $18.38 \pm 0.27$ \\
\hline $8 c$ & $5.66 \pm 0.47$ & $11.62 \pm 0.58$ & $17.28 \pm 1.73$ & $6.12 \pm 0.47$ & $10.68 \pm 0.58$ & $15.62 \pm 1.16$ \\
\hline $8 d$ & $7.26 \pm 0.58$ & $14.00 \pm 2.00$ & $21.86 \pm 1.53$ & $7.38 \pm 0.27$ & $14.26 \pm 0.58$ & $20.66 \pm 0.58$ \\
\hline $9 a$ & $7.68 \pm 0.58$ & $14.46 \pm 1.16$ & $21.68 \pm 1.16$ & $6.68 \pm 0.58$ & $12.57 \pm 0.58$ & $20.48 \pm 1.73$ \\
\hline $9 b$ & $8.14 \pm 0.47$ & $14.75 \pm 0.58$ & $21.72 \pm 1.16$ & $7.14 \pm 0.58$ & $12.44 \pm 0.58$ & $20.96 \pm 0.58$ \\
\hline $9 \mathrm{c}$ & $6.14 \pm 0.61$ & $11.62 \pm 0.58$ & $17.36 \pm 0.58$ & $5.84 \pm 1.16$ & $10.74 \pm 1.53$ & $17.85 \pm 0.27$ \\
\hline $9 d$ & $8.82 \pm 0.58$ & $16.58 \pm 0.58$ & $23.76 \pm 1.53$ & $7.36 \pm 0.58$ & $13.00 \pm 0.00$ & $22.66 \pm 0.58$ \\
\hline $10 \mathrm{a}$ & $5.44 \pm 0.58$ & $9.26 \pm 0.58$ & $17.16 \pm 1.16$ & $5.38 \pm 1.73$ & $10.12 \pm 0.61$ & $16.94 \pm 0.61$ \\
\hline $10 \mathrm{~b}$ & $5.76 \pm 0.58$ & $9.38 \pm 0.47$ & $18.00 \pm 2.00$ & $5.16 \pm 0.47$ & $9.18 \pm 0.58$ & $17.36 \pm 1.53$ \\
\hline $10 \mathrm{~d}$ & $6.12 \pm 0.58$ & $10.83 \pm 0.61$ & $19.47 \pm 1.53$ & $5.62 \pm 0.58$ & $10.86 \pm 0.61$ & $18.74 \pm 0.58$ \\
\hline $11 \mathrm{a}$ & $4.35 \pm 0.58$ & $8.72 \pm 1.16$ & $16.73 \pm 1.16$ & $4.57 \pm 2.08$ & $9.24 \pm 1.16$ & $15.26 \pm 2.08$ \\
\hline $11 \mathrm{~b}$ & $4.69 \pm 0.58$ & $9.16 \pm 2.08$ & $16.16 \pm 1.16$ & $4.93 \pm 0.58$ & $8.58 \pm 1.16$ & $15.78 \pm 1.53$ \\
\hline $11 \mathrm{~d}$ & $5.14 \pm 0.58$ & $9.86 \pm 1.16$ & $17.14 \pm 1.53$ & $5.85 \pm 0.58$ & $11.00 \pm 2.00$ & $16.42 \pm 0.58$ \\
\hline $12 \mathrm{a}$ & $5.86 \pm 0.58$ & $11.48 \pm 1.16$ & $17.47 \pm 1.53$ & $5.18 \pm 0.58$ & $11.12 \pm 0.17$ & $16.94 \pm 0.58$ \\
\hline $12 b$ & $6.10 \pm 0.58$ & $11.24 \pm 0.47$ & $17.22 \pm 1.53$ & $5.24 \pm 0.58$ & $10.62 \pm 0.27$ & $17.12 \pm 0.58$ \\
\hline $12 d$ & $6.38 \pm 0.58$ & $13.37 \pm 0.27$ & $19.72 \pm 0.27$ & $5.64 \pm 0.58$ & $13.86 \pm 0.61$ & $19.16 \pm 1.16$ \\
\hline $13 d$ & $4.72 \pm 0.27$ & $7.37 \pm 1.73$ & $12.46 \pm 1.16$ & $4.82 \pm 0.58$ & $9.38 \pm 0.61$ & $14.35 \pm 0.27$ \\
\hline $14 \mathrm{a}$ & $6.72 \pm 0.47$ & $13.85 \pm 0.61$ & $19.06 \pm 0.61$ & $6.41 \pm 0.58$ & $12.35 \pm 0.47$ & $18.49 \pm 0.58$ \\
\hline $14 \mathrm{~b}$ & $6.88 \pm 1.73$ & $12.69 \pm 0.58$ & $19.28 \pm 0.27$ & $6.05 \pm 0.58$ & $13.06 \pm 0.27$ & $17.35 \pm 1.53$ \\
\hline $14 \mathrm{c}$ & $5.14 \pm 0.61$ & $10.17 \pm 0.27$ & $16.36 \pm 0.47$ & $4.76 \pm 1.73$ & $8.49 \pm 1.16$ & $14.06 \pm 0.61$ \\
\hline $14 d$ & $7.96 \pm 0.58$ & $14.00 \pm 1.00$ & $20.31 \pm 1.16$ & $8.16 \pm 0.58$ & $15.72 \pm 0.47$ & $19.84 \pm 0.58$ \\
\hline $15 \mathrm{~d}$ & $5.62 \pm 0.58$ & $9.38 \pm 0.61$ & $16.49 \pm 0.27$ & $6.86 \pm 0.58$ & $9.63 \pm 1.73$ & $17.54 \pm 0.58$ \\
\hline $16 a$ & $7.36 \pm 0.61$ & $11.92 \pm 1.16$ & $18.17 \pm 1.53$ & $7.46 \pm 0.47$ & $12.15 \pm 0.61$ & $18.12 \pm 0.58$ \\
\hline $16 \mathrm{~b}$ & $7.42 \pm 0.58$ & $11.88 \pm 0.47$ & $19.02 \pm 0.27$ & $7.56 \pm 0.58$ & $13.00 \pm 0.00$ & $18.30 \pm 0.27$ \\
\hline $16 \mathrm{c}$ & $5.84 \pm 0.27$ & $9.63 \pm 1.73$ & $16.47 \pm 0.58$ & $5.63 \pm 0.561$ & $11.37 \pm 0.27$ & $15.10 \pm 0.61$ \\
\hline $16 d$ & $8.72 \pm 0.47$ & $13.96 \pm 1.16$ & $21.36 \pm 0.61$ & $7.85 \pm 0.27$ & $13.94 \pm 0.61$ & $20.82 \pm 0.27$ \\
\hline Griseofu & ulvin - & & $24.67 \pm 1.16$ & $-\quad-$ & $23.14 \pm 2.00$ & \\
\hline
\end{tabular}

${ }^{*} \mathrm{SD}=$ Standard Deviation; 1 = Candida albicans; 2 = Aspergillus niger 
Table 4. Antibacterial activity of compounds.

$\frac{\text { Table 4. Antibacterial activity of compounds. }}{E . c o l i}$

\begin{tabular}{|c|c|c|c|c|c|c|c|c|c|c|c|c|}
\hline \multirow[b]{2}{*}{$\overline{\text { Compc }}$} & \multicolumn{3}{|c|}{ E.coli ${ }^{I}$} & \multicolumn{2}{|c|}{$P$. aeruginosa $^{2}$} & \multicolumn{4}{|c|}{ B. subtilis ${ }^{3}$} & \multicolumn{3}{|c|}{ S. aureus ${ }^{4}$} \\
\hline & $\begin{array}{l}\mathrm{d} \begin{array}{l}10 \mu \mathrm{g} / \mathrm{mL} \\
\pm \mathrm{SD}^{*}\end{array}\end{array}$ & $\begin{array}{c}20 \mu \mathrm{g} / \mathrm{mL} \\
\pm \mathrm{SD}^{*}\end{array}$ & $\begin{array}{c}30 \mu \mathrm{g} / \mathrm{mL} \\
\pm \mathrm{SD}^{*}\end{array}$ & $\begin{array}{c}10 \mu \mathrm{g} / \mathrm{mL} \\
\pm \mathrm{SD}^{*}\end{array}$ & $\begin{array}{c}20 \mu \mathrm{g} / \mathrm{mL} \\
\pm \mathrm{SD}^{*}\end{array}$ & $\begin{array}{c}30 \mu \mathrm{g} / \mathrm{mL} \\
\pm \mathrm{SD}^{*}\end{array}$ & $\begin{array}{r}10 \mu \mathrm{g} / \mathrm{mL} \\
\pm \mathrm{SD}^{*}\end{array}$ & $\begin{array}{c}20 \mu \mathrm{g} / \mathrm{mL} \\
\pm \mathrm{SD}^{*}\end{array}$ & $\begin{array}{c}30 \mu \mathrm{g} / \mathrm{mL} \\
\pm \mathrm{SD}^{*}\end{array}$ & $\begin{array}{c}10 \mu \mathrm{g} / \mathrm{mL} \\
\pm \mathrm{SD}^{*}\end{array}$ & $\begin{array}{l}20 \mu \mathrm{g} / \mathrm{mL} \\
\pm \mathrm{SD}^{*}\end{array}$ & $\begin{array}{c}30 \mu \mathrm{g} / \mathrm{mL} \\
\pm \mathrm{SD}^{*} \\
\end{array}$ \\
\hline $4 a$ & $6.86 \pm 1.73$ & $11.72 \pm 2.52$ & $16.52 \pm 2.65$ & $6.72 \pm 1.16$ & $11.38 \pm 0.58$ & $15.49 \pm 0.58$ & $.82 \pm 0.47$ & $11.15 \pm 1.16$ & $16.16 \pm 1.53$ & $5.82 \pm 0.58$ & & \\
\hline $4 b$ & $6.08 \pm 0.58$ & $.28 \pm 0.47$ & & $92 \pm 0.61$ & $85 \pm 0$. & & $6 \pm 0.58$ & & & & & 0.58 \\
\hline $4 c$ & $5.16 \pm 1.16$ & $84 \pm 1.53$ & $4.18 \pm 2.52$ & $.10 \pm 0.58$ & $.63 \pm 0.5$ & & $0 \pm 0.58$ & $9.02 \pm 1$ & & & 1.16 & $16.60=$ \\
\hline $4 d$ & & \pm 0.58 & & \pm 0.26 & & & & & & & & 0.58 \\
\hline $5 \mathrm{a}$ & & $=2.52$ & & & & & & & & & & \\
\hline $5 b$ & $5.25 \pm 1.15$ & $0 \pm 0.00$ & & $6 \pm 0.57$ & & & $9 \pm .57$ & & & & .57 & .57 \\
\hline $5 c$ & $5.84 \pm 0.58$ & $19 \pm 0.58$ & 53 & $67 \pm 0.45$ & \pm 0 & & $8 \pm 1.16$ & 9 & 1.73 & & $1.29 \pm 0.61$ & 2.00 \\
\hline & & \pm 0.58 & & \pm 0.00 & & & & & & & & .26 \\
\hline oa & & \pm 0.58 & & & & & & & & & & .58 \\
\hline $6 b$ & $7.85 \pm 1.73$ & \pm 2.52 & & & & & & & & & & \\
\hline $6 c$ & $7.22 \pm 1.1 .6$ & $.96 \pm 0.47$ & 21 & $6.90 \pm 0.58$ & 6 & & & 12.5 & & & & 0.26 \\
\hline $6 \mathrm{~d}$ & $8.74 \pm 0.58$ & $.27 \pm 1.16$ & 58 & $8.17 \pm 2$. & 17 & & 53 & 45 & & & $14.10 \pm 1.16$ & 0.58 \\
\hline & & 17 & & & & & & & & & & .26 \\
\hline $8 \mathrm{~b}$ & & \pm 1.16 & & & & & & & & & & \\
\hline $8 c$ & & $.73 \pm 1.16$ & & 6 & 9 & & & & & & & .61 \\
\hline $8 d$ & & \pm 0.47 & 17 & \pm 0.61 & & & 17 & & & & .17 & 0.58 \\
\hline & & & & & & & & & & & & .17 \\
\hline $9 \mathrm{~b}$ & & \pm 0.47 & & 0.17 & 99 & & & & & & & .17 \\
\hline $9 \mathrm{c}$ & & & & & & & & & & & & 1.73 \\
\hline $9 d$ & $8.54 \pm 0.47$ & $.27 \pm 0.58$ & 20.79 & $39 \pm 1.53$ & 45 & & 1.16 & & & & \pm 2.09 & 20.17 \\
\hline & & & & & & & & & & & 1.16 & \\
\hline $14 b$ & & $17 \pm 2.09$ & 16 & \pm 1.17 & 58 & & & & & & 0.61 & .26 \\
\hline $14 c$ & & $.27 \pm 0.45$ & & & & & & & & & \pm 0.47 & 0.17 \\
\hline $14 d$ & $7.37 \pm 0.61$ & $.89 \pm 0.58$ & 1.16 & $7.38 \pm 1.73$ & $.48 \pm 0.58$ & & & 13.60 & 0.61 & & \pm 0.58 & 19.81 \\
\hline & & $.18 \pm 0.63$ & & $6.60 \pm 1.53$ & & & & & & & & 0.58 \\
\hline $16 b$ & & & & & & & & & & & & \\
\hline $16 \mathrm{c}$ & $6.10 \pm 0.58$ & $.90 \pm 1.16$ & & $5.56 \pm 0.47$ & & & & & & & $11.47 \pm 0.58$ & $17.94 \pm 1.73$ \\
\hline $16 \mathrm{~d}$ & $7.12 \pm 0.26$ & $13.36 \pm 0.58$ & $19.26 \pm 0.47$ & $7.19 \pm 0.45$ & $13.28 \pm 0.17$ & & $7.31 \pm 0.58$ & $13.27 \pm 0$. & \pm 1.53 & & $12.90 \pm 2.09$ & $18.15 \pm 0.26$ \\
\hline & $5.78 \pm 1.53$ & $11.02 \pm 2.09$ & $16.85 \pm 1.16$ & $6.12 \pm 1.53$ & $11.82 \pm 0.58$ & $17.14 \pm 0.58$ & & & & & $11.51 \pm 0.47$ & $=0.58$ \\
\hline & mycin - & & - & & & - & - & - & $23.33 \pm 1.53$ & - & & \\
\hline & 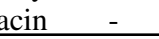 & & $22.67 \pm 0.58$ & & & $24.00 \pm-1.0$ & & & & & & \\
\hline
\end{tabular}

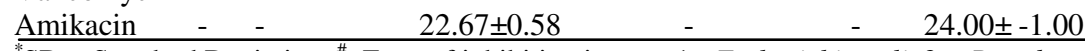

"SD = Standard Deviation; ${ }^{\#}$ Zone of inhibition in mm; $1=$ Escherichia coli $; 2=$ Pseudomonas aeruginosa $; 3=$ Staphylococcus aureus; $4=$ Bacillus subtilis 


\section{Analgesic activity}

All the compounds were tested for their analgesic activity using Eddy's hot plate technique ${ }^{17}$. Mice (Swiss strain) of either sex weighing between 25-35g were used for the experiment. In this method heat is used as a source of pain. Animals were individually placed on a hot plate maintained at constant temperature $\left(55^{\circ} \mathrm{C}\right)$ and the reaction of animals, such as paw licking or jump response (whichever appears first) was taken as the end point. A cut off - time of 15 seconds (sec) was taken as maximum analgesic response to avoid injury to the paws. Tested compounds and diclofenac sodium (standard) at a dose of $5 \mathrm{mg} / \mathrm{kg}$ body weight in $1 \%$ gum acacia was given as suspension orally to animals and observed the reaction time of animals on the hot plate at $15,30,60,90$ and 120 minutes (min) after the compound administration. Percentage analgesic activity shown by the tested compounds is recorded in Table 2.

Antibacterial and antifungal activities

Applying the agar plate diffusion technique ${ }^{18}$ all of the newly synthesized compounds were screened in vitro for antibacterial activity against Escherichia coli (E.coli), Pseudomonas aeruginosa (P. aeruginosa) (Gram negative), Staphylococcus aureus (S.aureus), Bacillus Subtilis (B.subtilis) (Gram positive) at $10 \mu \mathrm{g} / \mathrm{mL}$, $20 \mu \mathrm{g} / \mathrm{mL}, 30 \mu \mathrm{g} / \mathrm{mL}$ concentrations respectively. Under identical conditions the positive control antibiotics Amikacin at $30 \mu \mathrm{g} / \mathrm{mL}$ showed zone of inhibition 23-24mm for gram-negative organism and Vancomycin at $100 \mu \mathrm{g} / \mathrm{mL}$ showed zone of inhibition $23 \mathrm{~mm}$ for gram-positive organism. Similarly, the antifungal screening of the compounds were carried out in vitro by paper disc method against two fungai Aspergillus niger (A.niger) and Candida albicans (C.albicans) by using Griseofulvin $(30 \mu \mathrm{g} / \mathrm{mL})$ as the positive control, which showed (23mm and $25 \mathrm{~mm}$ respectively) as the zone of inhibition.

\section{Acknowledgements}

The authors are thankful to IISc, Bangalore, IIT, Chennai and STIC, Cochin, for the Spectral analysis. The authors are also thankful to the Chairman, Dept. of Chemistry, Kuvempu University, for providing laboratory facilities.

\section{References}

1 Colanceska-Ragenovic K, Dimova1 V, Kakurinov V, Labor D and Molnar A B, Molecules, 2001, 6, 815.

2 Labanauskas L, Udrenaite E, Gaidelis P and Bruk tus A, IL Farmaco, 2004, 59, 255.

3 Al-Soud Y A, Al-Dweri M N and Al-Masoudi N A, IL Farmaco, 2004, 59, 775.

4 Foroumadi A, Mirzaei M and Shafiee A, IL Farmaco, 2001, 56,621.

5 Jain S K and Mishra P, Ind.J.Chem., 2004, 43B, 184.

6 Swamy S N, Basappa B S, Prabhuswamy P B, Doreswamy B H, Prasad J S and Rangappa K S, European journal of Med. Chem., 2006, 41, 531.

7 Wang Z, You T, Yu Xu Haijian and Haoxin S Molecules, 1996, 1, 68.

8 Udupi R H, Kushnoor A and Bhat A R, J. Ind. Chem. Soc., 1999, 76, 461.

9 Gupta R, Sudan S and Kachroo P L, Ind. J. Chem., 1984, 23B, 793.

10 Gupta R, Satya Paul, Gupta A K, Kachroo P L and Bani S Ind. J. Chem., 1998, 37B, 498.

11 Hirpara H M, Sodha V A, Trivedi A M, Khatri B L and Parikh A R Ind. J. Chem., 2003, 42B, 1756.

12 Joshi K C and Giri S, J. Ind. Chem. Soc., 1963, 40, 42.

13 Haglind J. Chemical Abstract, 1966, 64,16509.

14 Reid J R and Heindel N D, J. Heterocycl. Chem., 1976, 13, 925.

15 Winter C A, Risley E A and Nuss G W, Proc. Soc. Exp. Biol. Med., 1962, 111, 544.

16 Kulkarni S K Handbook of Experimental Pharmacology; Vallabh Publications, 1999, $3^{\text {rd }}$ Ed., 128.

17 Eddy N B and Leimbach D J, J. Pharmacol. Exptl. Therap., 1953, 385.

18 Verma R S and Imam S A, Ind. J. Microbiol., 1973,13,45. 


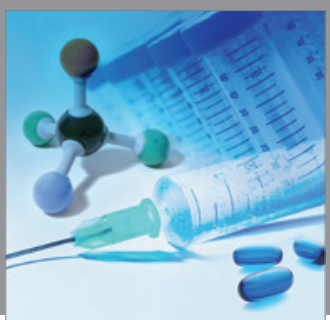

International Journal of

Medicinal Chemistry

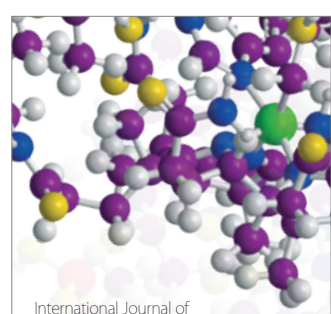

Carbohydrate Chemistry

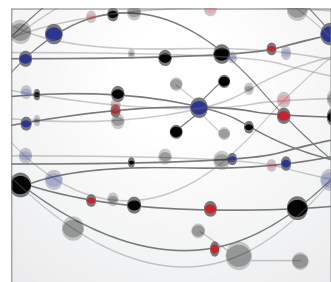

The Scientific World Journal
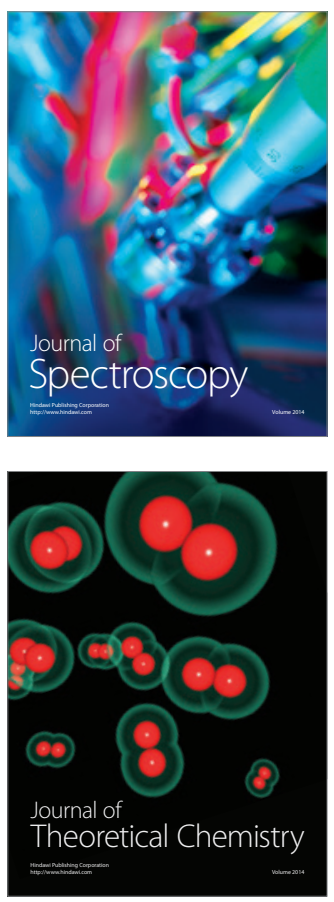
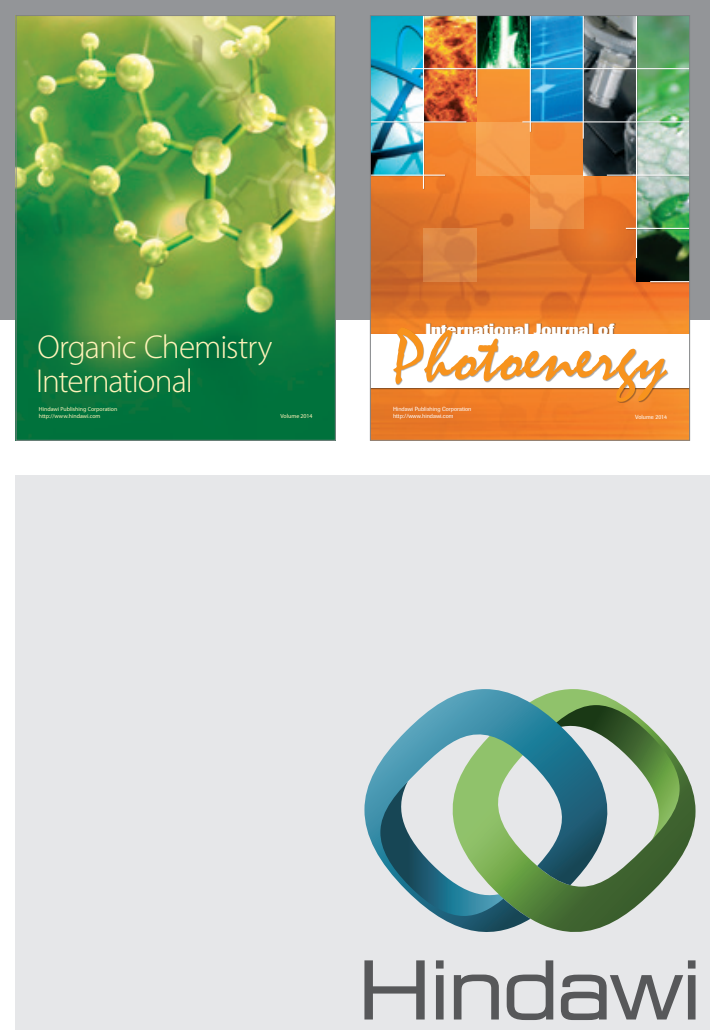

Submit your manuscripts at

http://www.hindawi.com
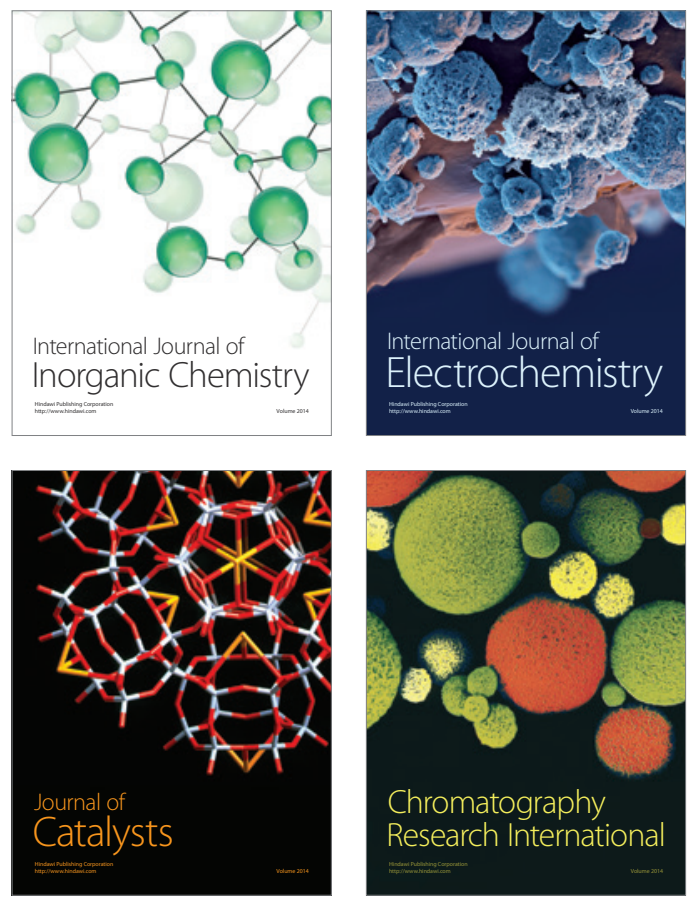
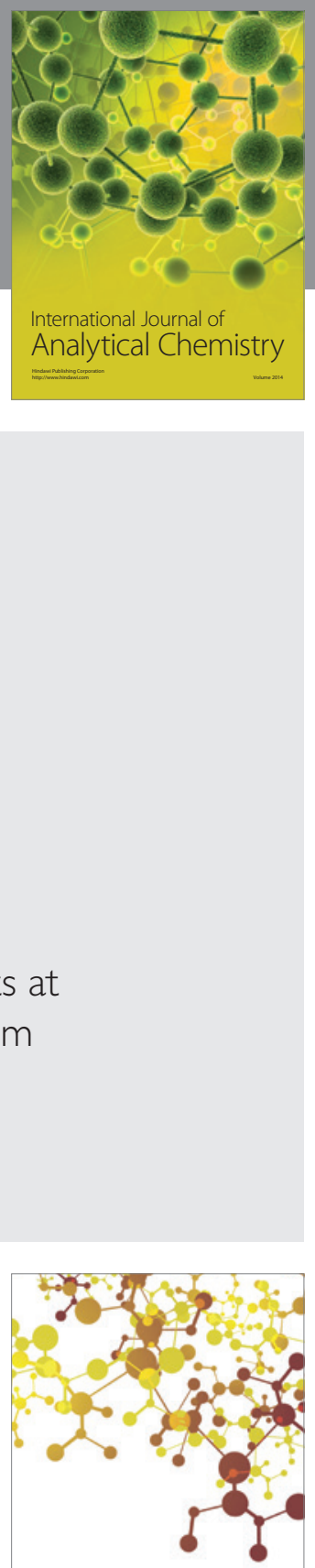

Journal of

Applied Chemistry
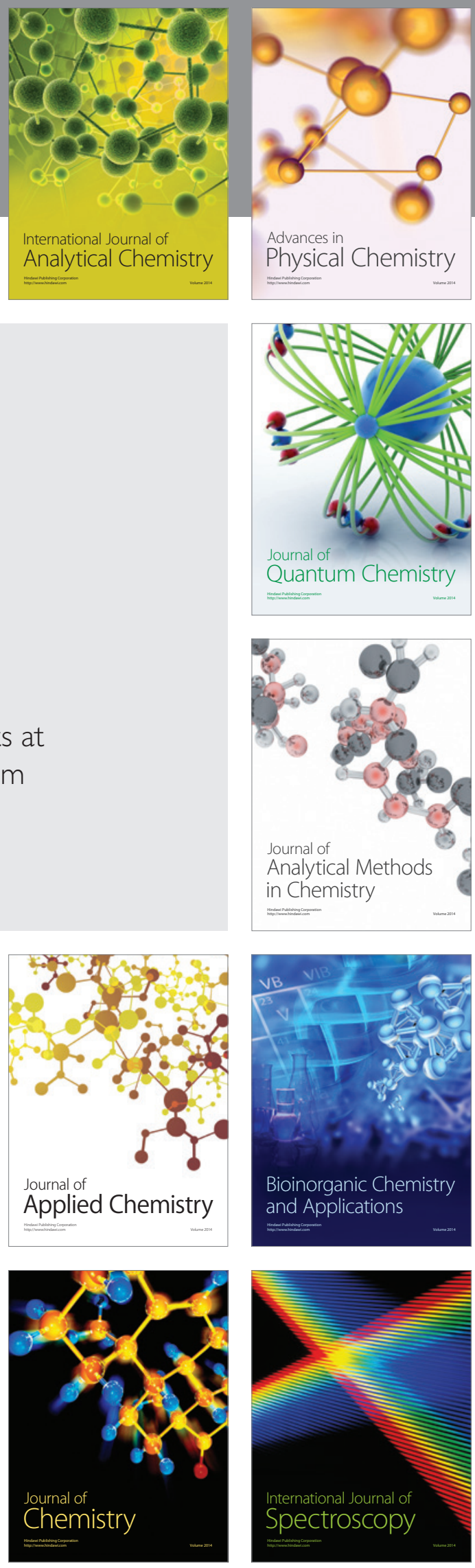